\title{
Evocation and characterization of percepts of apparent motion on the face
}

\author{
JULI A. SZANISZLO \\ University of Texas Health Science Center, San Antonio, Texas \\ and \\ GREG K. ESSICK, DOUGLAS G. KELLY, AARON K. JOSEPH, and KATHY R. BREDEHOEFT \\ University of North Carolina, Chapel Hill, North Carolina
}

\begin{abstract}
The percepts evoked by sequential stimulation of sites in close spatial proximity $(\leq 2.5 \mathrm{~cm})$ on the face were studied. Both method-of-limits and magnitude-estimation procedures were used to identify and characterize alterations in the percepts produced by systematic changes in the temporal and spatial parameters of the sequence. Each site was stimulated by a vertically oriented row of miniature vibrating probes. Apparent motion was consistently perceived when the delay between the onsets of sequentially activated rows (interstimulus onset interval, or ISOI) fell within a relatively narrow range of values, the lower limit of which approximated $5 \mathrm{msec}$. Both the upper limit and the perceived smoothness and continuity of the motion percepts (goodness of motion) increased with the duration for which each row stimulated the skin over the range evaluated, 15-185 msec. For the successive activation of only two rows, goodness of motion was not influenced by changes in their separation from 0.4 to $2.5 \mathrm{~cm}$. The ISOI values at which magnitude estimates of goodness of motion were highest increased with the duration for which each row stimulated the skin. As such, maximum goodness of motion decreased with increases in the apparent velocity of motion. When the number of sequentially activated rows was increased from two to four or more, the quality of the motion percepts improved. For the successive activation of multiple closely spaced rows, values of ISOI at which numerical estimates of goodness of motion were highest approximated integral fractions of the duration for which each row stimulated the skin. In this situation, the probes rose and fell in a regular, step-locked rhythm to simulate an edge-like or rectangular object moving across the skin. The goodness of motion so attained was relatively independent of the apparent velocity of motion.
\end{abstract}

The movement of a natural object across the skin evokes a rich perceptual experience and one that is acutely sensitive to subtle changes in the physical parameters of stimulation (see, e.g., Essick, Afferica, et al., 1988; Essick, Dolan, Turvey, Kelly, \& Whitsel, 1990; Essick, Whitsel, Dolan, \& Kelly, 1989). The richness of the percept is hypothesized to reflect, in part, the complexity of the stimulation. For example, a moving brush stimulus not only translates across but also laterally stretches the skin. The two mechanical actions (translation and stretch) generate unique stresses and strains, to which peripheral neural encoding mechanisms are differentially sensitive (Edin, Essick, Trulsson, \& Olsson, 1995).

This investigation was supported by Grants DE07509, 2-S07-RR 05333-29, and DE07160 from the National Institutes of Health, Bethesda, MD 20892. Stimulator research and development were funded by the University of North Carolina School of Dentistry. The authors thank Mike Young and Sunil Patel for preparation of the figures. J.A.S., now Juli A. Powell, is a dentist in Austin, TX. D.G.K. is in the Department of Statistics at the University of North Carolina. A.K.J. is a dermatologist in Houston, TX. Correspondence concerning this article should be addressed to G. K. Essick, Department of Prosthodontics and Curriculum in Neurobiology, 110 Dental Research Center, CB 7455, University of North Carolina, Chapel Hill, NC 27599-7455 (e-mail: greg_essick@) dentistry.unc.edu).
To better understand tactile motion perception, we studied the percepts evoked by stimuli that elicit response patterns in the cutaneous mechanoreceptors that are much simpler than those elicited by a natural moving stimulus (see Essick, Rath, Kelly, James, \& Murray, 1996). These stimuli were generated by a dense-array tactile stimulator that consisted of a matrix of independently controlled probes, each of which vibrated a small area of skin. With this device, a stimulus that translated across the skin was viewed as a unique spatiotemporal sequence of simpler nontranslating component stimuli. As such, the spatial location and duration of each component stimulus, as well as the temporal delay between the onsets of successively delivered component stimuli, could be systematically varied and the impact of the variations evaluated. Although the stimulus translated discontinuously across the skin without stretching it laterally, percepts of robust, smooth, and continuous motion could be attained.

The experiments reported in this paper sought to evaluate the percepts evoked by the stimuli delivered to the face. The primary aim was to identify those stimulus conditions that best simulate the translatory component of real movement across the skin for use in the study of trigeminal nerve-injured patients (see, e.g., Essick, 1992; Essick et al., 1996). With frictionless stimuli, information 
about direction of motion is anticipated to be available only from the spatiotemporal sequence of mechanoreceptor activation (Essick et al., 1996). As such, tests evaluating patients' ability to discriminate direction are predicted to be particularly useful in evaluating alteration in sensory function after nerve injury (Essick et al., 1990).

Our general approach was to systematically address the same questions that have been investigated by others who have studied the percepts evoked by discrete vibrotactile stimulation of two or more sites across the skin (see, e.g., Kirman, 1974a, 1974b, 1975, 1983; Sherrick, 1968; Sherrick \& Rogers, 1966; Sumby, 1955). Specifically, what, if any, temporal conditions (i.e., the delay between the onset of stimulation of successive sites and the duration of stimulation) support percepts of apparent motion (Experiments 1,2, and 3)? How are the temporal stimulus conditions and the frequency and quality of motion percepts impacted by the number of discrete sites stimulated (Experiments 2 and 3 )? To what extent can an effect of the number stimulated be attributed to the concomitant increase in the total length of skin stimulated (Experiments 2 and 3 )?

Earlier studies have demonstrated that percepts of motion can be evoked by the successive stimulation of only two skin sites. Moreover, changes in the spatial separation between the sites (from 0.5 to $5 \mathrm{~cm}$, Kirman, 1974a, $1974 \mathrm{~b}$; from 12 to $40 \mathrm{~cm}$, Sherrick, 1968; from 4 to $12 \mathrm{~cm}$, Sumby, 1955) do not impact either the temporal conditions for percepts of motion or their quality. In contrast, when more than two sites are successively stimulated, less time is required between their onsets of stimulation, and percepts of motion are evoked more frequently and with better quality (Kirman, 1974b, 1975). For two sites and more than two sites, motion percepts also increase in frequency and quality with the duration for which each skin site is stimulated (Kirman, 1974a, 1974b, 1975; see also Gibson, 1968). Moreover, the delay between the onset of stimulation of successive sites becomes less critical (Kirman, 1974a, 1974b, 1975, 1983). As will be made evident, the findings of the present study are wholly consistent with, and attest to the validity and generality of, these previous findings.

The present study differs notably, however, from other vibrotactile studies conducted over the past 30 years. First, no other investigation has systematically studied apparent motion evoked by stimuli applied to facial skin (but see Essick, McGuire, Joseph, \& Franzén, 1992). Although test-site location has been shown to have minimal impact on percepts of apparent motion (thigh, forearm, back, stomach, palm, and fingertip have all been studied; Kirman, 1974a; Sherrick, 1968), the receptor mechanisms in facial skin differ from those on sites studied previously-the most notable departure being the absence of the Pacinian channel (Barlow, 1987; Hollins, Delemos, \& Goble, 1991). The lack of this channel alone was hypothesized to result in a sharper localization of tactile sensation (Sherrick, Cholewiak, \& Collins, 1990), which, in turn, might lower the optimal delay between the onset of stimulation of successive sites for percepts of apparent motion (Kirman, 1974a). Second, the number of stimuli employed in the present study was appreciably greater, and the spacing between adjacent stimuli was less, than those of other investigations. Only the stimuli employed by Kirman (1983) resembled those employed in the present study. However, a maximum of 14 stimuli (compared to 22 in the present study) was sequentially delivered in his study, and the minimum spacing exceeded four times that of the dense-array tactile stimulator. Given even the closest spacing of stimuli employed by Kirman $(0.51 \mathrm{~cm}$; Kirman, 1974b, 1975, 1983) and the skin site to which they were delivered (fingers), successive stimuli did not, in general, activate the same peripheral receptive field. In contrast, in the present study, an edge or rectangular array composed of small vibrating probes could be used to simulate translation across the skin, with discontinuities approximating one-third or one-fourth the diameter of the cutaneous receptive fields of the midfacial region (Johansson, Trulsson, Olsson, \& Westberg, 1988; see also Appendix A). Third, in the present work, the same subjects were studied with two experimental approaches (modified method of limits and absolute magnitude estimation) to maximize and validate the information obtained. Previous investigators have employed either method-of-limits or adjustment procedures when stimulus timing variables were under study (Kirman, 1983; Sherrick, 1968; Sherrick \& Rogers, 1966) or magnitudeestimation or rating procedures when the subjective quality of the percepts was under study (Kirman, 1974a, 1974b, 1975; Sumby, 1955). Fourth, the same group of subjects was employed in all three experiments, thus eliminating the potential contribution of among-group differences to the observed effects. In contrast, past studies have typically employed different groups of subjects to address different questions. The additional insight into the evocation and character of percepts of apparent motion provided by our experiments has been reported previously in abbreviated format (Szaniszlo, Essick, \& Kelly, 1992).

\section{GENERAL METHOD}

\section{Subjects}

Four college students (three females and one male, ages 21, 22, 23 , and 29 years) with previous experience as psychophysical subjects consented to participate in the study. Approximately 30 half-day sessions were required of each subject. The study was approved by the Human Subjects Committee at the University of North Carolina School of Dentistry. Reimbursement was provided at $\$ 5.00 /$ hour. Two or three rest periods were offered during each testing session.

\footnotetext{
Apparatus

Tactile stimuli were provided by a novel dense-array tactile stimulator (see Essick et al., 1996, for a detailed description). Briefly described, the stimulator system consisted of a transducer module, driving electronics, and a computer. The transducer module consisted of 288 ( 24 rows $\times 12$ columns) 0.01 -in.-diameter metal probes
} 


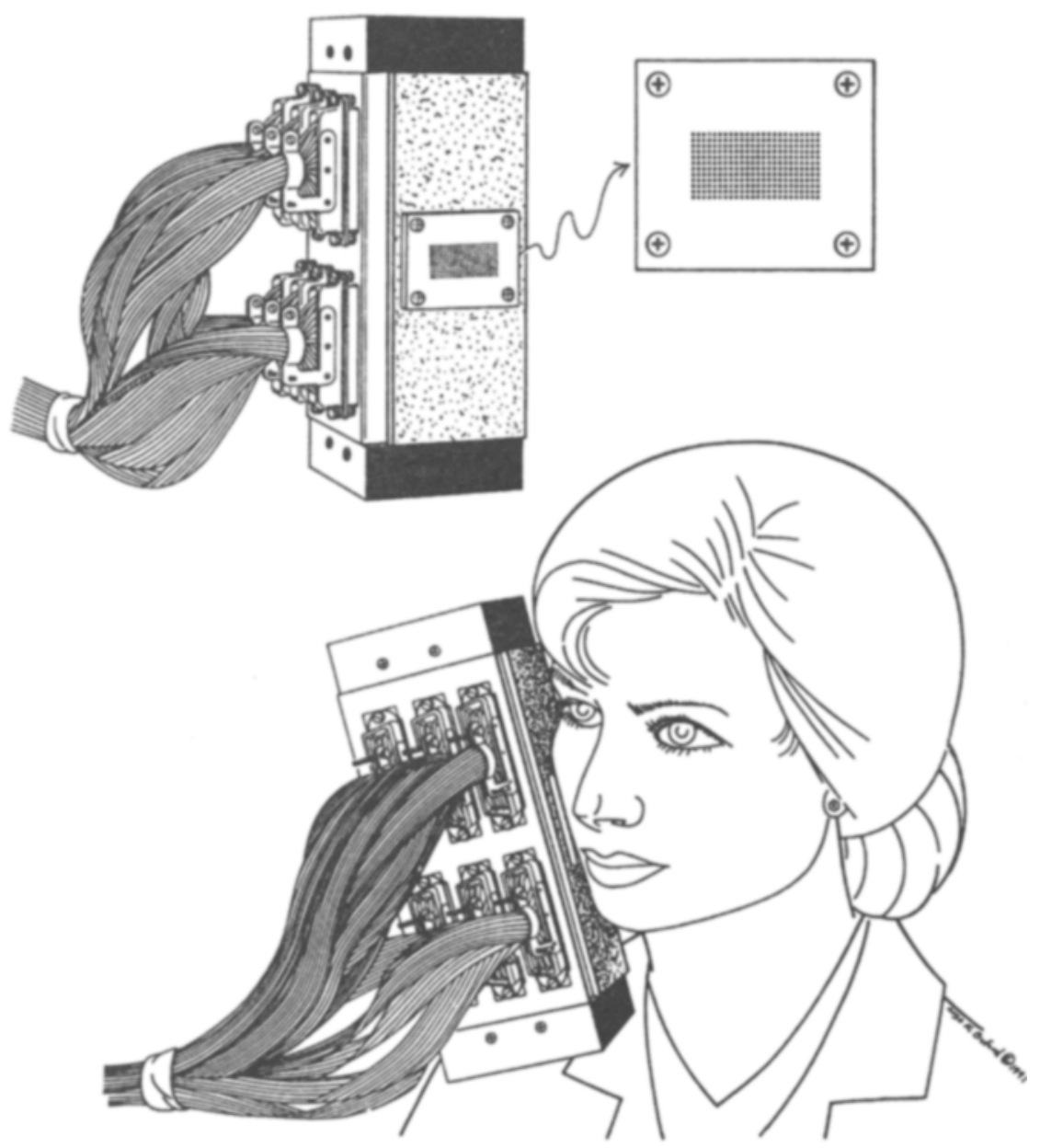

Figure 1. The transducer module of the dense-array tactile stimulator. (Top) Close-up sketch of array of 288 probes. (Bottom) Transducer positioned to stimulate skin of the midportion of the right cheek.

(Figure 1). The probe center-to-center was nominally $0.12 \mathrm{~cm}$ across the rows and columns, such that the total array was $2.7 \mathrm{~cm}$ long and $1.3 \mathrm{~cm}$ wide. The probes extended through an adjustable display plate covered with a thin commercially available plastic film, Saran Wrap (Dow Chemical Company). The film was replaced after each session in order to maintain cleanliness of the array of probes and to minimize transmission of skin bacteria among subjects (see also Cholewiak \& Collins, 1990). Each probe was attached to a piezoelectric bender/bimorph that deflected in proportion to an imposed voltage from 0 to $40 \mathrm{~V}$. For the experiments reported in this paper, selections of the 288 probes for activation (i.e., frames) were specified every $2.5 \mathrm{msec}$ in a manner that would generate vibrating edgelike patterns composed of rows. Thus, the active probes were driven by a square wave at $200 \mathrm{~Hz}$ with a duty cycle of $50 \%$. The period of time that each row was displayed (hereafter referred to as stimulus duration or duration of activation), as well as the time between the onset of activation of successive rows (hereafter referred to as the interstimulus onset interval [ISOI]), were restricted to multiples of $5 \mathrm{msec}$.

\section{Stimuli}

During each of three experiments, the percepts evoked by four different spatial patterns were studied (Figure 2). Each pattern consisted of the sequential activation of rows of the dense-array tactile stimulator. The impact of the rate at which the rows were sequen- tially activated (determined by the ISOI) and of their individual duration of activation was of major interest. Four different durations were studied: $15 \mathrm{msec}$ ( 3 square wave cycles), $35 \mathrm{msec}$ ( 7 cycles), $95 \mathrm{msec}$ ( 19 cycles), and $185 \mathrm{msec}(37$ cycles). No current was delivered to the probes during the final $2.5 \mathrm{msec}$ of the duration period. The apparent velocity of motion was defined as the distance between adjacent rows divided by the ISOI.

\section{Procedure}

Each subject was comfortably seated in a dimly lit, quiet room and faced a color monitor that cued him(her) as to trial initiation, stimulus delivery, and responses. Headphones, through which white noise was provided, were worn to eliminate sound cues. The transducer of the dense-array tactile stimulator was positioned in light contact with the middle of the right fleshy cheek during all testing (Figure 1). Contact with tissue directly overlying or firmly attached to bone was avoided. The array was positioned horizontally on the face such that the rows were oriented vertically (viz., superoinferiorally with respect to the head). The sequence of row activation was always in the lateral-to-medial (ear-to-nose) direction. The subject operated a three-button computer mouse to vary the stimulus parameters under study and/or to enter responses.

Each experiment was performed twice. During the first series of sessions for each experiment, a modified method of limits was employed. During the second series, absolute magnitude estimation was 


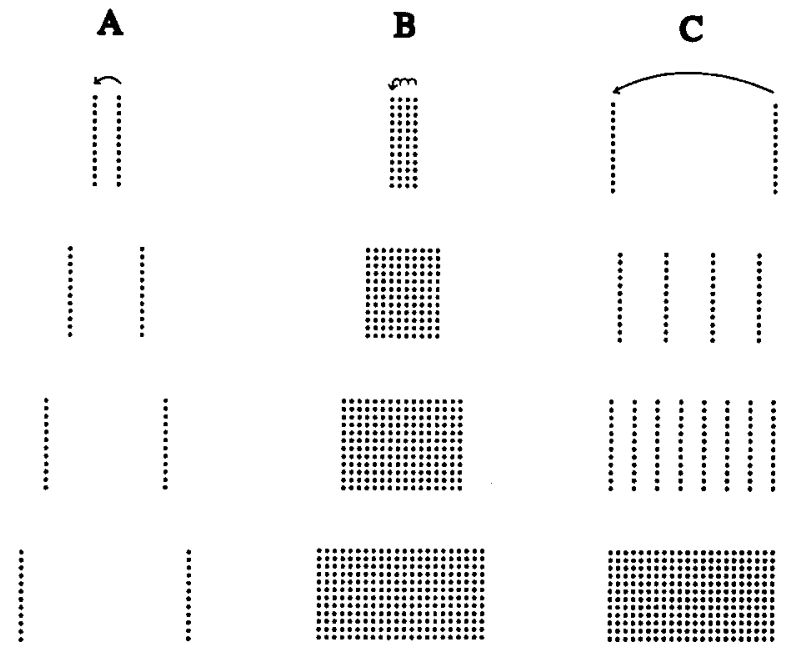

Figure 2. The spatial patterns studied in Experiment 1 (A), Experiment 2 (B), and Experiment 3 (C). In Experiment 1, only two rows, separated by one of four distances, were successively activated. In Experiment 2, closely spaced rows were sequentially activated over the separations employed in Experiment 1. In Experiment 3 , the total distance over which percepts were evoked was held constant. The number (density) of equally spaced rows defined the experimental variable. The arrows at the top indicate the order in which the rows were activated, as viewed from the transducer display plate (Figure 1, top).

employed. For all sessions, good motion was defined as a smooth, continuous, and uninterrupted translatory percept (see also below).

For each session during which the method of limits was employed, 4 of the 16 combinations of spatial pattern $\times$ stimulus duration were randomly chosen for study without replacement. Initially, the ISOI was set by the experimenter to a high value at which the presentation of each row (i.e., a "bumpy" succession of discrete stimuli rather than motion per se) was felt. A stimulus was delivered approximately every $3 \mathrm{sec}$. The subject was instructed to press the left mouse button repetitively to decrease the ISOI by 5 -msec-long intervals until the rows were displayed simultaneously (ISOI $=0$ ) and to comment on changes in the percepts as they occurred. The descending series of stimulation was followed by an ascending series: The subject increased the ISOI, initially at $0 \mathrm{msec}$, by $5 \mathrm{msec}$ with each press of the right mouse button. Comment markers were entered into the data file by pressing the middle mouse button. The experimenter recorded on paper the actual comments made by the subject, which were later coded and inserted into the data file. Within the range of ISOI values supporting percepts of motion, the subject was instructed to alternatively decrease and increase the ISOI in order to locate values at which the motion felt particularly smooth and continuous. Practice was provided before any data were collected. Specifically, the subjects were instructed to first study the percepts evoked during a descending and ascending series of stimulation. Data were then collected during a second (descending and ascending) series. Using this approach, approximately $30 \mathrm{~min}$ were required to obtain data for each combination of spatial pattern $x$ stimulus duration. Before data were collected, driving voltages were identified by one highly trained subject for the stimuli at which all of the combinations of spatial pattern $\times$ stimulus duration could be easily felt but at which no stimulus was uncomfortably intense.

The magnitude-estimation protocol in the present study was similar to that described by Gibson (1968), who used ratio scaling to a standard to obtain estimates of the amplitude of perceived movement evoked by electrical stimuli applied to the back. In the present study, however, a variant of free magnitude estimation without a standard or modulus (viz., absolute magnitude estimation; see Collins \& Gescheider, 1989; Gescheider, 1988) was employed. The stimulus for each trial consisted of one combination of the four spatial patterns, the four stimulus durations, and 50 values of ISOI that ranged from 5 to $250 \mathrm{msec}$. In total, 1,390 unique spatial/temporal patterns were defined. The subject was required to evaluate the percept evoked by each pattern on $\mathbf{4}$ different days.

During each session, the stimuli were delivered in random order with regard to spatial pattern, duration, and ISOI. At the end of each trial, the subject scaled the goodness of motion and was told to ignore variations in intensity, velocity, and distance traveled. Extensive preliminary experimentation revealed that subjects associated the goodness-of-motion attribute evoked by the dense-array stimuli with perceived smoothness and continuity rather than with less tangible qualities, such as robustness or impressiveness. Since we were also interested in those spatial and temporal stimulus conditions that evoked percepts similar to those evoked by a constant-velocity natural stimulus moving continuously across the skin, the following instructions were written and read at the beginning of every session:

After feeling each stimulus, you are asked to estimate the magnitude of the goodness-of-motion. In other words, answer how smooth and continuous the motion felt. To do this, first decide whether or not the stimulus felt like motion. If it did not, use the computer mouse to enter the lowest possible value of "l." If it did feel like motion, assign a number to the stimulus in such a manner that your impression of how large the number is matches your impression of how good (smooth and continuous) the motion felt. You may use any positive number you deem appropriate, except "1," which indicates no motion. Do not let what you feel or how you respond on any trial affect how you respond on any subsequent trial.

The left and right mouse buttons decreased and increased, respectively, the integer shown to the subject on the computer monitor. The rate at which the numbers changed increased with the duration for which the buttons were depressed. The subject entered the number shown on the monitor by pressing the middle mouse button. Since the perceived intensities of the stimuli would have varied greatly had they been delivered with the same driving voltage, their tactile loudness was first adjusted to a criterion level by one highly trained subject. This was accomplished with a two-alternative, forced choice paradigm by which the voltages for the test stimuli were systematically varied (see Appendix to Essick et al., 1996).

\section{Statistical Approach}

The impact of all experimentally varied temporal and spatial parameters on indices that summarized the subjects' responses was assessed by repeated-measures analysis-of-variance procedures. Specifically, an $F$ statistic for determining the significance of the effect of each parameter on each index was calculated by dividing the parameter mean square by the subject-by-parameter mean square. The subject-by-parameter mean square assessed the extent to which the parameter differentially influenced different subjects' percepts and was replaced by the mean squared residual for calculating $F$ in those analyses for which the interaction was insignificant ( $p$ values $>.05$; Myers, 1966, pp. 152-173). The indices that were selected for analysis are described with each experiment below. For magnitude-estimation sessions, the intraclass correlation coefficient (Koch, 1985) was also calculated in order to assess the consistency with which the experimental variables influenced subjects' responses on day-to-day replication of the stimuli.

\section{EXPERIMENT 1 Two Rows, Different Spatial Separations}

\section{Method}

The purpose of the first experiment was to determine whether percepts of apparent motion could be evoked by the successive stimulation of two sites in close spatial proximity on the face and 
whether the temporal and spatial stimulus conditions for these percepts were similar to those reported for other body regions. Each stimulus consisted of the successive activation of two rows of probes (Figure 2A). The four patterns differed 7 -fold in the spatial separation between the rows (viz., $0.4 \mathrm{~cm}, 1.1 \mathrm{~cm}, 1.8 \mathrm{~cm}$, and $2.5 \mathrm{~cm}$ ). For values of ISOI exceeding the stimulus duration, activation of the two rows was separated temporally by a positive interval of time. For ISOIs equal to the stimulus duration, the second row was activated precisely $2.5 \mathrm{msec}$ after the final current pulse to the first row. For values of ISOI less than the stimulus duration, a period existed during which both rows were simultaneously activated.

\section{Results and Discussion}

Method-of-limits sessions. Each subject identified the range of ISOI values over which motion was perceived. The highest value of ISOI supporting percepts of motion increased with stimulus duration $[F(3,9)=21.5, p<$ .0003 ; Figure 3, left panel]. For the shortest duration stimuli $(15 \mathrm{msec})$, the highest value averaged $41 \mathrm{msec}$, indicating that it was not necessary that these brief stimuli overlap in time for motion to be perceived. In contrast, for the longest duration stimuli $(185 \mathrm{msec})$, the highest value of ISOI averaged $154 \mathrm{msec}$, indicating that minimum temporal overlap was requisite for percepts of motion. Similar observations were made by Sherrick and Rogers (1966, p. 177) for stimuli that were separated by distances 10 to 100 times those employed in this experiment. The highest value of ISOI was also affected by the spatial separation between the two rows $[F(3,27)=3.32$, $p<.04]$. For the widely separated rows $(2.5 \mathrm{~cm})$ and longest duration tested $(185 \mathrm{msec})$, the highest value was slightly lower than that for separations $\leq 1.8 \mathrm{~cm}$ (see Figure 3, left panel). Moreover, the highest values marked during the descending series averaged $7.8 \mathrm{msec}$ less than the highest values marked during the ascending series $[F(1,27)=7.35, p<.02]$, which suggests that subjects were slightly conservative in indicating change in the qualitative nature of the percepts.

In contrast to the case for the highest value of ISOI, motion was consistently perceived for the lowest positive value $(5 \mathrm{msec})$. However, for at least one series of stimulation for 3 of the 4 subjects, a short gap in the range of ISOI values was identified over which the percepts did not meet their criteria for motion. This occurred on only 8 of the 128 series and merits no further consideration.
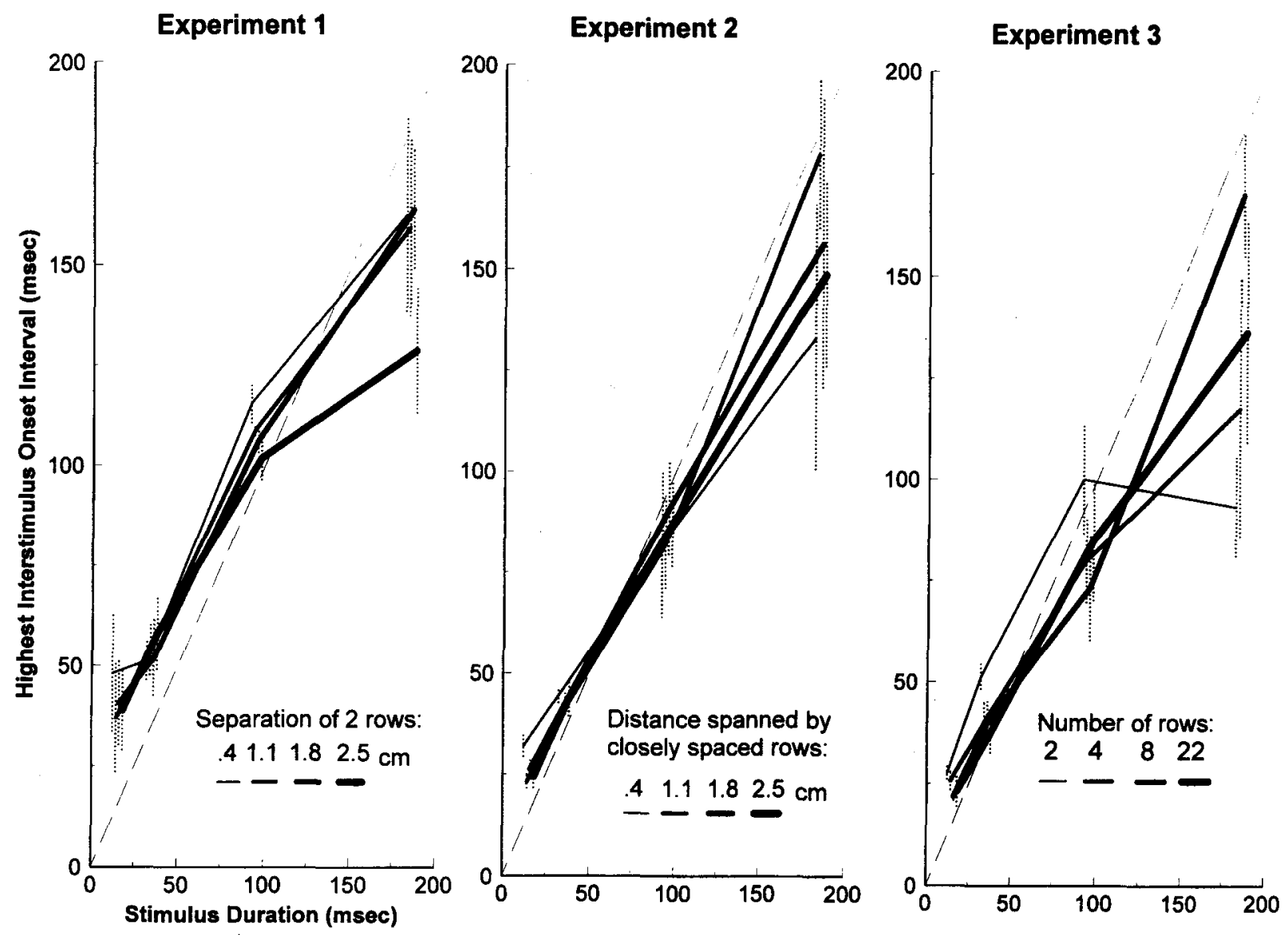

Figure 3. Highest interstimulus onset interval values supporting percepts of motion, from the method-of-limits data of Experiment 1 (left panel), Experiment 2 (middle panel), and Experiment 3 (right panel). Means, \pm 1 standard error, for different spatial patterns have been offset for clarity of presentation. The diagonal dashed lines denote reference lines of equality (i.e., $y$-values $=$ $x$-values). 
In addition to the identification of the range of ISOI values supporting motion percepts, the subjects identified values at which the percepts were particularly smooth and continuous. An ISOI value was defined as a local optimum if the motion percepts evoked by both slightly higher and slightly lower values were not as smooth and continuous. The number of optima marked by the subjects increased, on average, from 1.1 for the shortest duration stimuli to 2.5 for the longest duration stimuli, independently of the spatial separation of the two rows. The frequency at which each value of ISOI was marked as an optimum is shown in Figure 4 (top row of plots). With few exceptions, every value within the range of ISOI values supporting motion percepts was marked at least once. This confirms that smooth and continuous motion was perceived over the entire range of ISOI values identified. It also attests to the error in this experimental approach for the identification of optimal values for apparent motion. The limits defined by the mean optimal value $\pm 1 S D$ have been shown in previous work to approximate or exceed those defined by the highest and lowest ISOI values (Sherrick, 1968). As a result, some investigations have taken the midpoint of the interval between the highest and lowest ISOI value to represent an optimal value (Rogers, 1964). We alternatively employed a magnitude-estimation approach to determine the optimal value.

Magnitude-estimation sessions. The intraclass correlation coefficient (mean $=.72$ ) confirmed a high degree of within-subjects response consistency. Geometric means were calculated for each combination of row separation $\times$ stimulus duration and were plotted as functions of ISOI in order to generate the curves shown in Figure 5. Three observations were readily made. First, the plots are characterized by an inverted U-shaped form abutting the $y$-axis, which suggests that motion was perceived over a range of ISOI values, the lower limit of which approximated the lowest value tested $(5 \mathrm{msec})$. Second, stimulus duration had a profound effect on the goodness of motion (height of curve) as well as on the range of ISOI values supporting percepts of motion (width of curve). Third, the spatial separation between the two successively activated rows did not notably affect either the goodness of motion or the range of ISOI values over which percepts of motion were evoked.

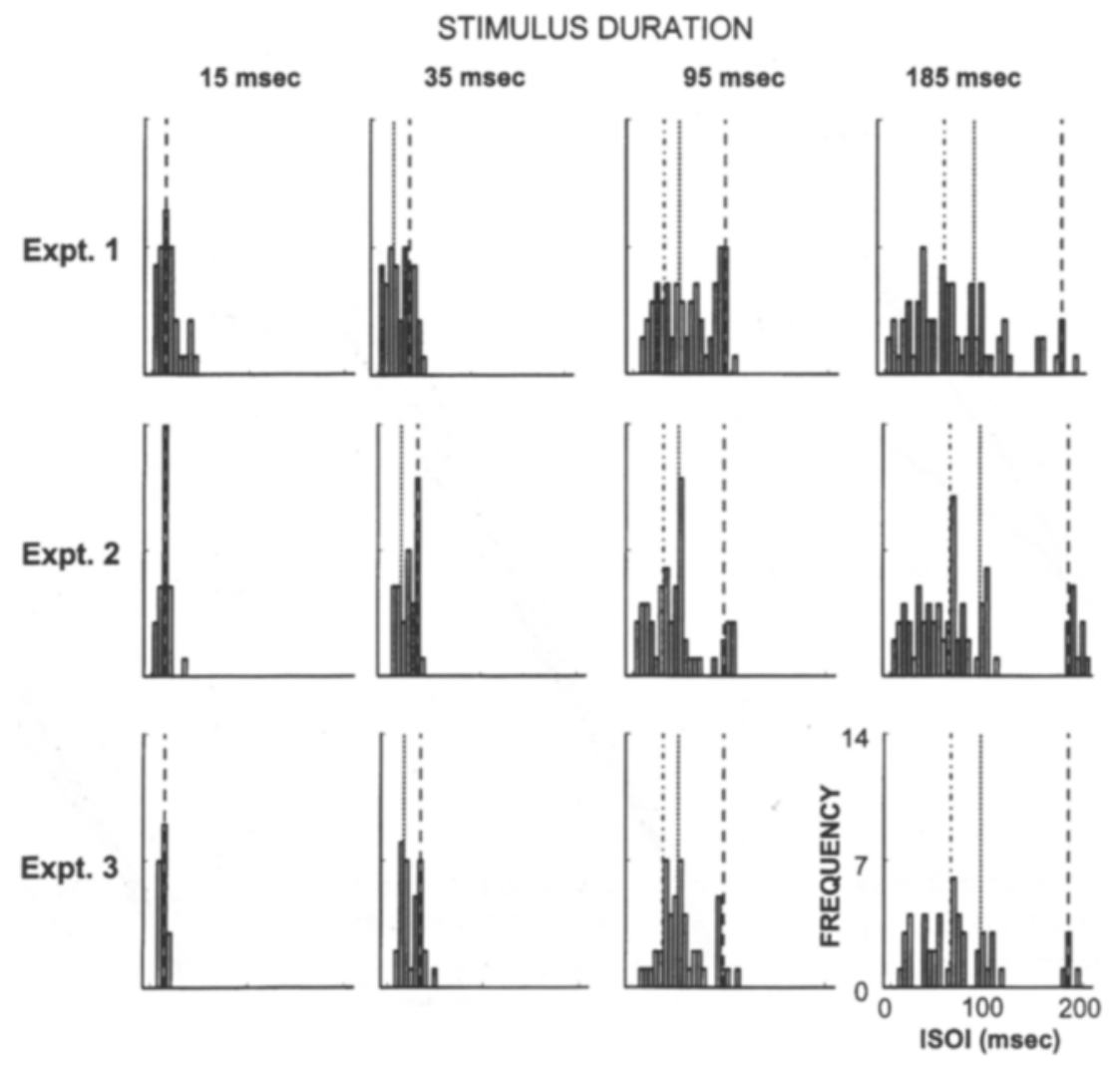

Figure 4. Frequency for which each value of the interstimulus onset interval (ISOI) was marked as an optimum in method-of-limits sessions. Each column of plots depicts data obtained with stimuli of one duration; each row, data from one of the three experiments. The data from all 4 subjects for all four spatial patterns were pooled to generate each plot. The vertical lines demarcate reference ISOI values at the first (dashed lines), second (dotted lines), and third (dot-dashed lines) integral fractions of the duration of stimulation. 


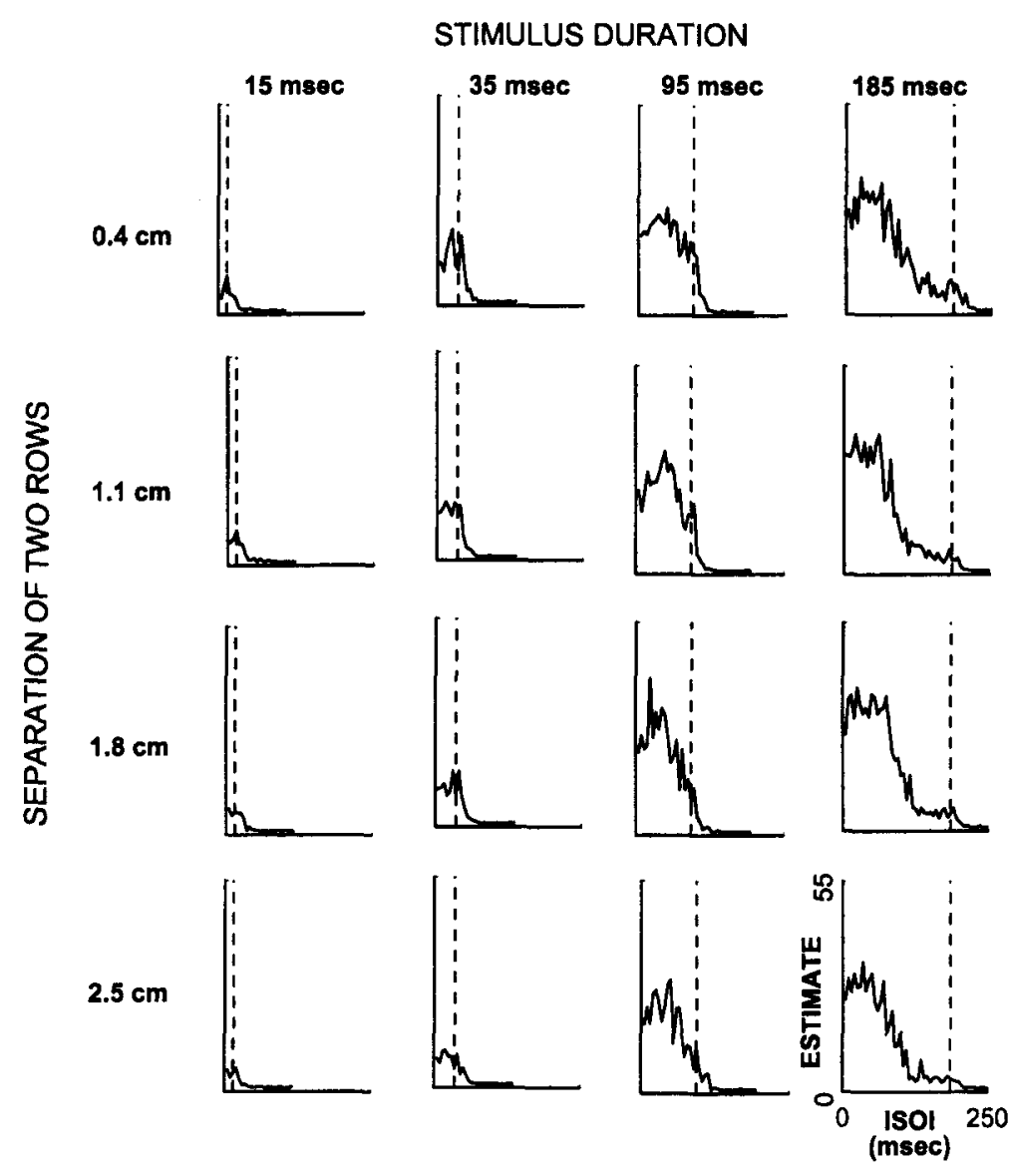

Figure 5. Geometric means of the magnitude estimates from Experiment 1, plotted as a function of interstimulus onset interval (ISOI) for each duration of stimulation (column of plots) and for each value of separation between the two successively activated rows (row of plots). The dashed vertical lines demarcate reference ISOI values equal to the duration of stimulation.

To statistically evaluate these subjective observations, the mean responses from each subject were plotted, and six measures were extracted, as illustrated in Figure 6.

1. The highest value of ISOI at which motion percepts were attained. This measure was calculated as the largest value of ISOI at which the magnitude estimate equaled $5 \%$ of its maximum.

2. The lowest value of ISOI at which motion percepts were attained. This measure was calculated as the smallest value of ISOI at which the magnitude estimate equaled $5 \%$ of its maximum or was set to $5 \mathrm{msec}$ if the magnitude estimate exceeded this criterion at ISOI $=5 \mathrm{msec}$.

3 . The range of ISOI values supporting motion percepts (from 1 and 2 above).

4. The highest magnitude estimate ( $y$-axis value) provided by the subject with regard to the smoothness and continuity of motion, defining the maximum goodness of motion.

5 . The ISOI value ( $x$-axis value) at which the highest magnitude estimate occurred, defining the optimal ISOI value.
6 . The area under the curve that graphically represents the magnitude estimate versus ISOI relationship between the high and low ISOI limits.

The results of the analyses of the highest, the lowest, and the range of ISOI values that supported motion percepts were remarkably similar to those of analogous measures extracted from the method-of-limits data. To illustrate, the highest mean ISOI value increased from 24 to $176 \mathrm{msec}$ with the increase in stimulus duration from 15 to $185 \mathrm{msec}$. The lowest ISOI value averaged $5.47 \mathrm{msec}$ and varied insignificantly with the stimulus duration and the spatial separation between the two rows.

The last three measures defined above were unique to the magnitude-estimation data and extended the characterization of the motion percepts. Most notably, the highest magnitude estimate was unaffected by the spatial separation between the two rows $[F(3,9)=1.01, p>.43]$, but increased 5.2-fold from the shortest to the longest stimulus duration $[F(3,9)=7.67, p<.008$; Figure 7 , left panel]. This indicates that maximum goodness of motion improved subjectively over five times with stimulus du- 


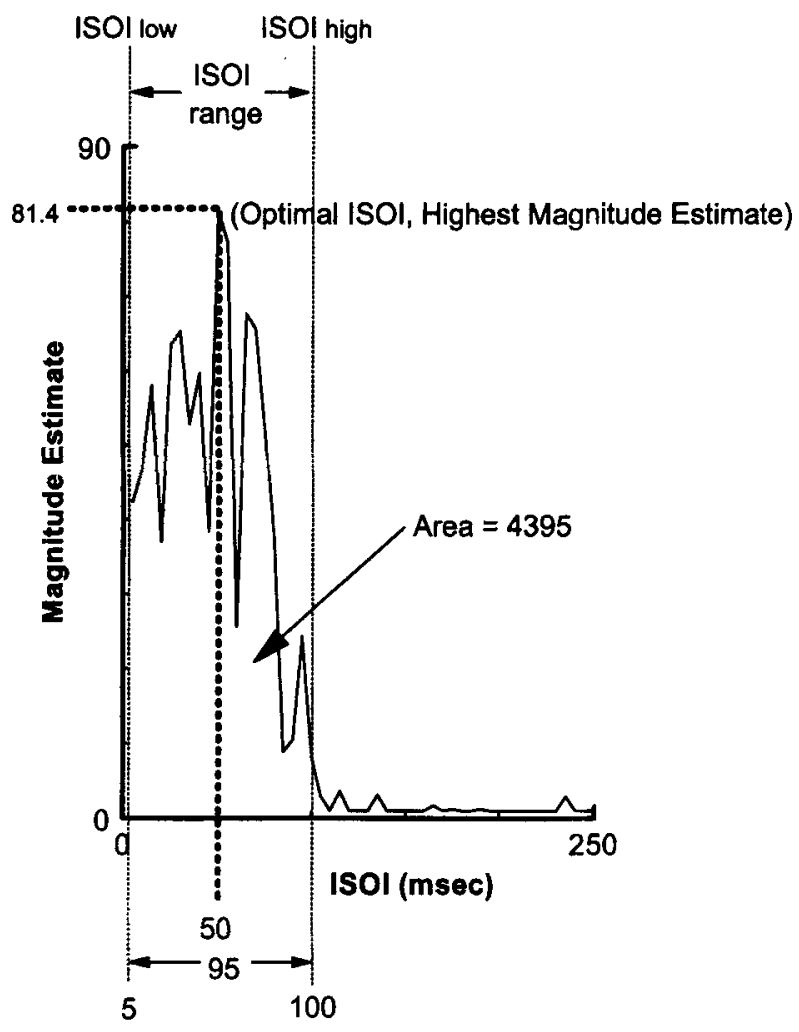

Figure 6. Geometric means of the magnitude estimates from Subject 224 when the two rows were separated by $1.8 \mathrm{~cm}$ and stimulus duration equaled $185 \mathrm{msec}$. The relationship between the goodness of motion and interstimulus onset interval was characterized by the six measures indicated (see text).

ration, assuming that the subjects employed ratio scales. Only a small portion of the improvement, however, was observed between the two longest stimulus durations, which suggests that variation in duration was most critical when it was less than $95 \mathrm{msec}$.

The ISOI value at which the maximum goodness of motion occurred also increased with stimulus duration $[F(3,9)=5.83, p<.02$; Figure 7 , middle panel $]$. The relationships depicted are similar to those observed by Sherrick and Rogers (1966, p. 178) and by Kirman (1974a, pp. 3-4); however, the values of the present study are lower. Kirman (1974a) argued that lower values can be attributed to increased sharpness of stimulus localization rather than to other parameters, such as the distance between skin sites. At least three factors in the present study may have contributed to a particularly high degree of sharpness in stimulus localization: (1) a probe diameter, one-half the size of the smallest employed in previous work (Kirman, 1974a); (2) the use of square-wave pulses to drive the probes (Kirman, 1974a); and (3) the absence of the Pacinian channel, which imparts the quality of diffuse hum to percepts evoked by vibrotactile stimuli (Barlow, 1987; Hollins et al., 1991; Sherrick et al., 1990).
Sharpness of localization, however, was not evaluated per se.

In addition to a main effect of stimulus duration on the optimal values of ISOI, a significant spatial separation $\times$ stimulus duration interaction was detected $[F(9,27)=$ $2.41, p<.04]$, but it accounted for only $14 \%$ of the total variability in the data. Averaging revealed that the optimal ISOI value approximated $60 \mathrm{msec}$ for stimuli of duration $\geq 95 \mathrm{msec}$. Given the relatively stable quality of the motion percepts for stimulus duration $\geq 95 \mathrm{msec}$ (Figure 7, left panel), goodness of motion improved little for values of duration and ISOI that exceeded 95 and $60 \mathrm{msec}$, respectively. Previous studies that have found higher optimal values of ISOI (cf. paragraph above) have similarly suggested that the frequency at which motion percepts are evoked and their subjective quality increase little for stimulus durations greater than $150 \mathrm{msec}$ at the optimal value of ISOI (ca. $100 \mathrm{msec}$; Kirman, 1974a).

The data shown in Figure 7 (left and middle panels) enabled calculation of the apparent velocities achieved by the full range of optimal temporal stimulus conditions. The velocities observed with the four separations are identified by the range of $x$-values over which the regression lines in Figure 8, left panel, have been drawn. The ordinate of the plot predicts the best motion that can be attained on a relative scale, calculated from the magnitude estimates provided by the subjects. Only for the shortest separation between two stimuli $(0.4 \mathrm{~cm})$ did the range of apparent velocities (viz., 1.8 to $35 \mathrm{~cm} / \mathrm{sec}$ ) coincide with the range useful for studying direction discrimination on the face $(0.5$ to $32 \mathrm{~cm} / \mathrm{sec}$; Essick, Afferica, et al., 1988). Moreover, as suggested by the negative slopes of the regression lines, the best percepts of apparent motion decreased in quality with increases in apparent velocity $[F(1,56)=13.2, p<.05]$. The figure indicates that one would need to vary the spatial separation between two rows rather than the temporal parameters of stimulation to experimentally attain a criterion level of goodness of motion at a given apparent velocity.

The final measure extracted from the magnitude-estimation data, area, estimated the total sum of the magnitude estimates over the range of ISOI values for which motion was perceived. Area was uninfluenced by the spatial separation of the two rows $[F(3,9)=2.09, p>.17]$ but increased 14.2-fold from the shortest to longest stimulus duration $[F(3,9)=6.94, p<.02$; Figure 7 , right panel]. That area increased with stimulus duration to an extent three times that of the highest magnitude estimate is consistent with Figure 5: As the stimulus duration increased, the magnitude estimates not only increased in general but over broader ranges of ISOIs. These observations suggest that the percept of motion evoked by two briefly presented rows was not particularly good, even when the second row was activated after an optimal delay. In contrast, with longer durations of activation, not only was the goodness of motion appreciably better, but 

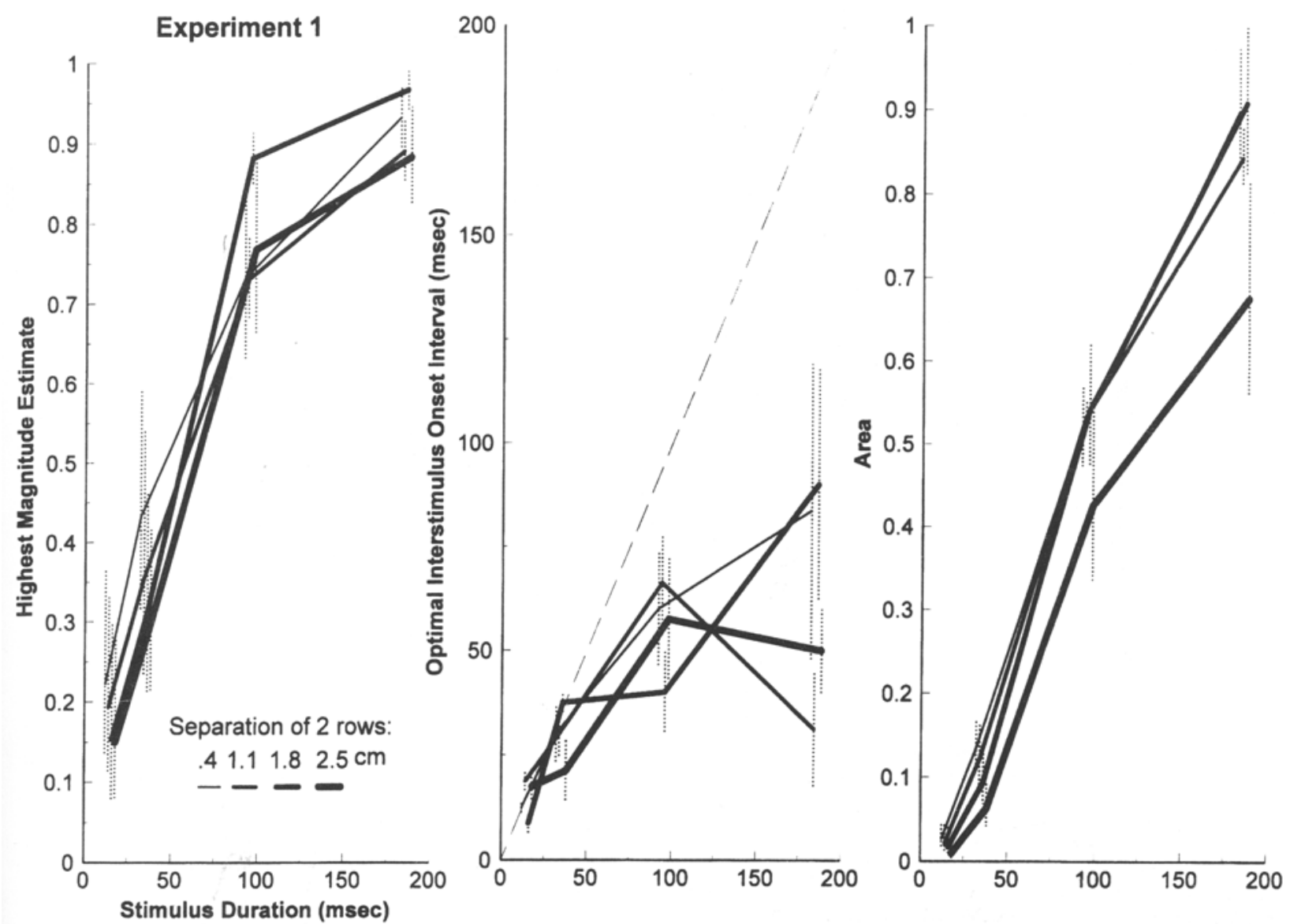

Figure 7. The highest magnitude estimates of goodness of motion (left panel), interstimulus onset interval (ISOI) values at which the highest estimates occurred (middle panel), and the area under the magnitude estimate versus ISOI relationship (right panel) obtained from the data for Experiment 1 . Means, \pm 1 standard error $(n=4$ subjects), for different spatial patterns have been offset for clarity of presentation. Since the subjects used different ranges of numbers, the highest magnitude estimates (left panel) and areas (right panel) were first normalized by dividing each by the subject's maximum value.

the delay between activations of the two rows was less critical.

\section{EXPERIMENT 2}

\section{Adjacent Rows, Different Distances}

\section{Method}

In Experiment 1, the percepts evoked by two successively activated rows, varying 7 -fold in spatial separation, were studied. It was argued that these stimuli would not be useful in the study of direction discrimination of the translatory component of real movement across the facial skin. Specifically, the temporal conditions that resulted in smooth and continuous percepts of motion also resulted in apparent velocities that exceed the range over which human subjects extract useful information, including direction of motion (see Essick, Frenzén, \& Whitsel, 1988). In the second experiment, we sought to physically simulate the translatory component of real movement across the skin with the stimulator: $4,10,16$, and 22 closely spaced rows were sequentially activated (Figure $2 \mathrm{~B}$ ). The numbers of rows were selected so that the separations between the first and last rows would correspond to those employed in Experiment 1.

For the multirow patterns of Experiment 2, a positive time interval during which no probes were activated was present between the successive activation of every two rows when the ISOI exceeded the stimulus duration. In contrast to Experiment 1 , when the ISOI equaled the stimulus duration, the more medial row rose and the adjacent (lateral) row fell in a highly regular, step-locked rhythm to closely simulate a real edge-like object moving across the skin. More generally, when the ISOI equaled an integral fraction $(1 / N)$ of stimulus duration, the $(i+N)$ th row was activated $2.5 \mathrm{msec}$ after the final pulse to the $i$ th row. As such, a total of $N$ rows was activated at the same time, except at the initiation and termination of the stimulation, and the apparent velocity of movement was $N$ times that observed for $N=1$. Consequently, the moving edge widened to $N$ rows to emulate, for example, a brush that moved onto, across, then off the skin. Since these stimuli most closely resemble the natural stimuli that we employed in past studies of direction discrimination, we sought to evaluate the impact of stimulus velocity and distance traversed on the percepts evoked.

\section{Results and Discussion}

Method-of-limits sessions. In a manner similar to that in Experiment 1, the highest value of ISOI that supported percepts of motion increased with stimulus duration $[F(3,9)=23.1, p<.0002$; Figure 3 , middle panel $]$, although values more often fell below the diagonal line 

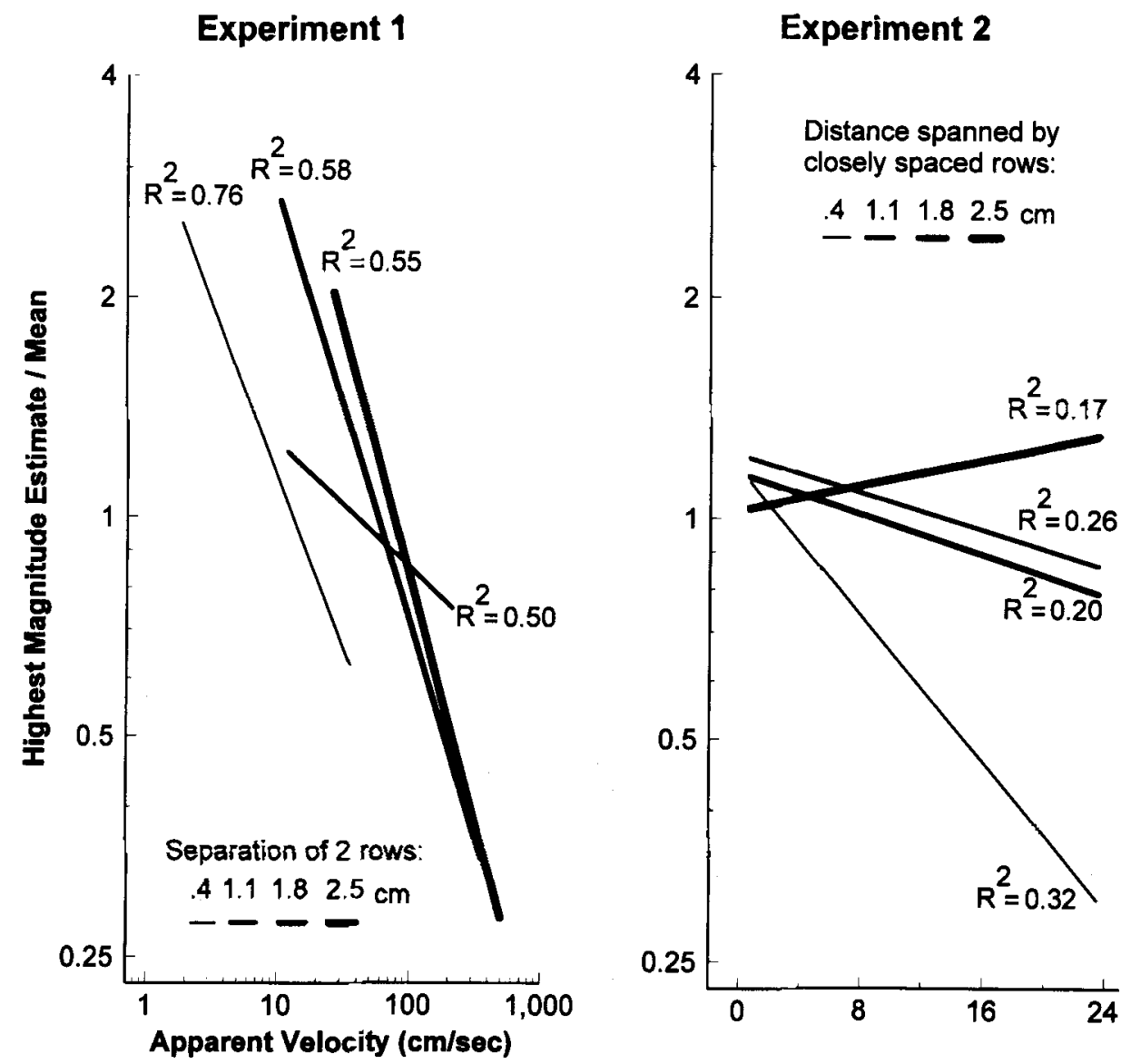

Figure 8. Relationships between highest magnitude estimates and apparent velocity of motion for Experiment 1 (left panel) and Experiment 2 (right panel). Only data from those temporal conditions that led to a smooth transition of one row to the next or to the simultaneous activation and translation of two (or three) rows were included in the analysis for Experiment 2. Regression lines were calculated after normalizing the highest magnitude estimates to each subject's average value. The range of $x$-values for each line indicates the range of apparent velocity over which the magnitude estimates were obtained. Note that both the $y$-axis and the $x$-axis (left panel only) are scaled logarithmically. $R^{2}$ values include model variance attributable to among-subjects differences in the slope and intercept.

of equality. This implies that, compared with the tworow patterns, temporal overlap in the activation of successive rows was more critical for the multirow patterns, particularly when stimulus duration exceeded $50 \mathrm{msec}$.

As in Experiment 1, motion was perceived for the lowest positive value of ISOI $(5 \mathrm{msec})$. Gaps in the range of ISOI values, over which the percepts did not meet criteria for motion, were identified by all of the subjects when the stimulus duration equaled $185 \mathrm{msec}$ (frequency = $56 \%$ ) and by 3 of the 4 subjects when the stimulus duration equaled $95 \mathrm{msec}$ (frequency $=28 \%$ ). Identification of gaps did not depend on the distance spanned by the stimuli, and gaps were identified for both descending and ascending series. The gaps typically included values of ISOI that were nonintegral fractions of stimulus duration. In this situation, the total number of rows activated at the same time alternated, causing the width of the simulated bar to oscillate as it translated across the skin. Gaps in the range of ISOI values that supported apparent motion have not been reported previously. It is likely that this is due to an insufficient number of values tested. To illustrate, Kirman (1983) employed only two and a half levels of ISOI shorter than the stimulus duration, on average, in studying patterns which consisted of up to 14 sequentially activated stimuli.

The number of optima marked by the subjects increased from 0.88 to 2.53 , on average, as stimulus duration increased from its shortest to its longest value, independently of the distance spanned. The frequency at which each value of ISOI was marked is shown in Figure 4 (second row of plots). The distributions appeared to exhibit peaks at ISOI values approximating the first three integral fractions of stimulus duration (i.e., one times, onehalf times, and one-third times the stimulus duration; see 


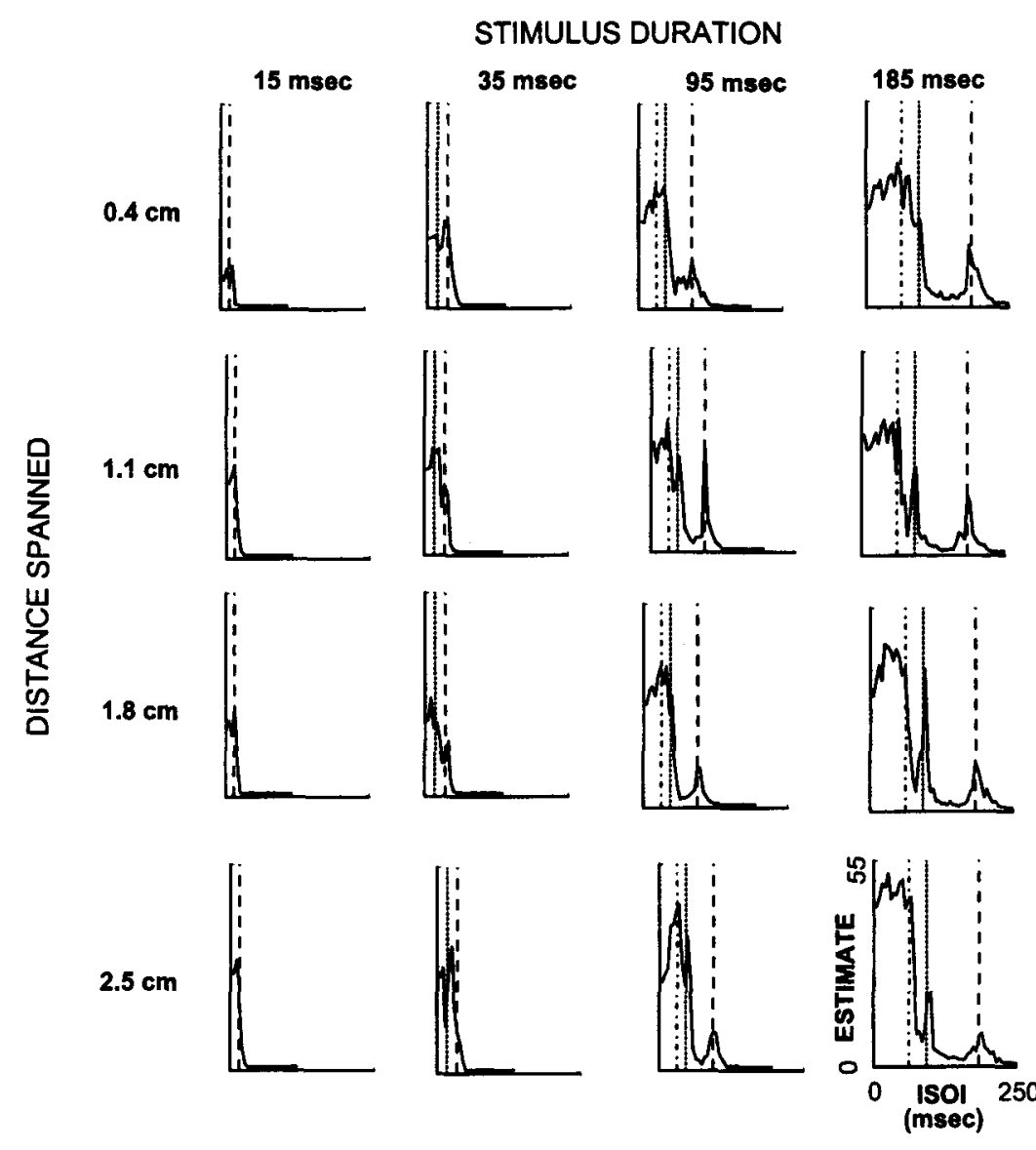

Figure 9. Geometric means of the magnitude estimates of Experiment 2, plotted as a function of interstimulus onset interval (ISOI) for each duration of stimulation (column of plots) and distance spanned across the skin (row of plots). The vertical lines demarcate reference ISOI values at the first (dashed lines), second (dotted lines), and third (dot-dashed lines) integral fractions of the duration of stimulation.

the plots for higher stimulus durations). That these values resulted in particularly good percepts of apparent motion was verified by the magnitude-estimation data.

Magnitude-estimation sessions. The intraclass correlation coefficient (mean $=.78$ ) confirmed a high degree of day-to-day consistency in the influence of the experimental variables on each subject's responses. The means of the magnitude estimates were plotted as functions of ISOI, as is shown in Figure 9. Four observations are readily made on inspection of the mean data. First, for the shorter durations at which the individual rows were activated ( 15 and $35 \mathrm{msec}$ ), the plots are notably similar to those in Experiment 1. Second, for the longer durations of row activation ( 95 and $185 \mathrm{msec}$ ), the plots are characterized not only by an inverted U-shaped form abutting the $y$-axis but by additional peaks at one times (third and fourth columns) and one-half times (fourth column) the stimulus duration. Thus, the peaks occurred at values of ISOI for which local optima were most commonly marked during the method-of-limits sessions (see paragraph above and Appendix B). Third, as in Experi- ment 1 , stimulus duration had a noted effect on the goodness of motion (height of peaks) as well as on the range of ISOI values that supported percepts of motion (total width of curve). Fourth, the total distance over which the stimuli were delivered appeared to affect the goodness of motion (unlike what was the case in Experiment 1) but not the total range of ISOI values over which the percepts were evoked. To statistically evaluate these subjective observations, the measures used to characterize the data from individual subjects in Experiment 1 were extracted and analyzed. Given that multiple peaks were observed in the profiles, AREA was not calculated, since its analysis would have been uninterpretable.

As in Experiment 1, the results of the highest, the lowest, and the range of ISOI values supporting motion percepts were similar to those of the analogous measures determined from the method-of-limits data. Two major differences between Experiments 1 and 2 were observed. First, the highest magnitude estimate increased with stimulus duration only 2.2-fold, as compared with 5.2fold in Experiment $1[F(3,9)=5.32, p<.03$; compare 

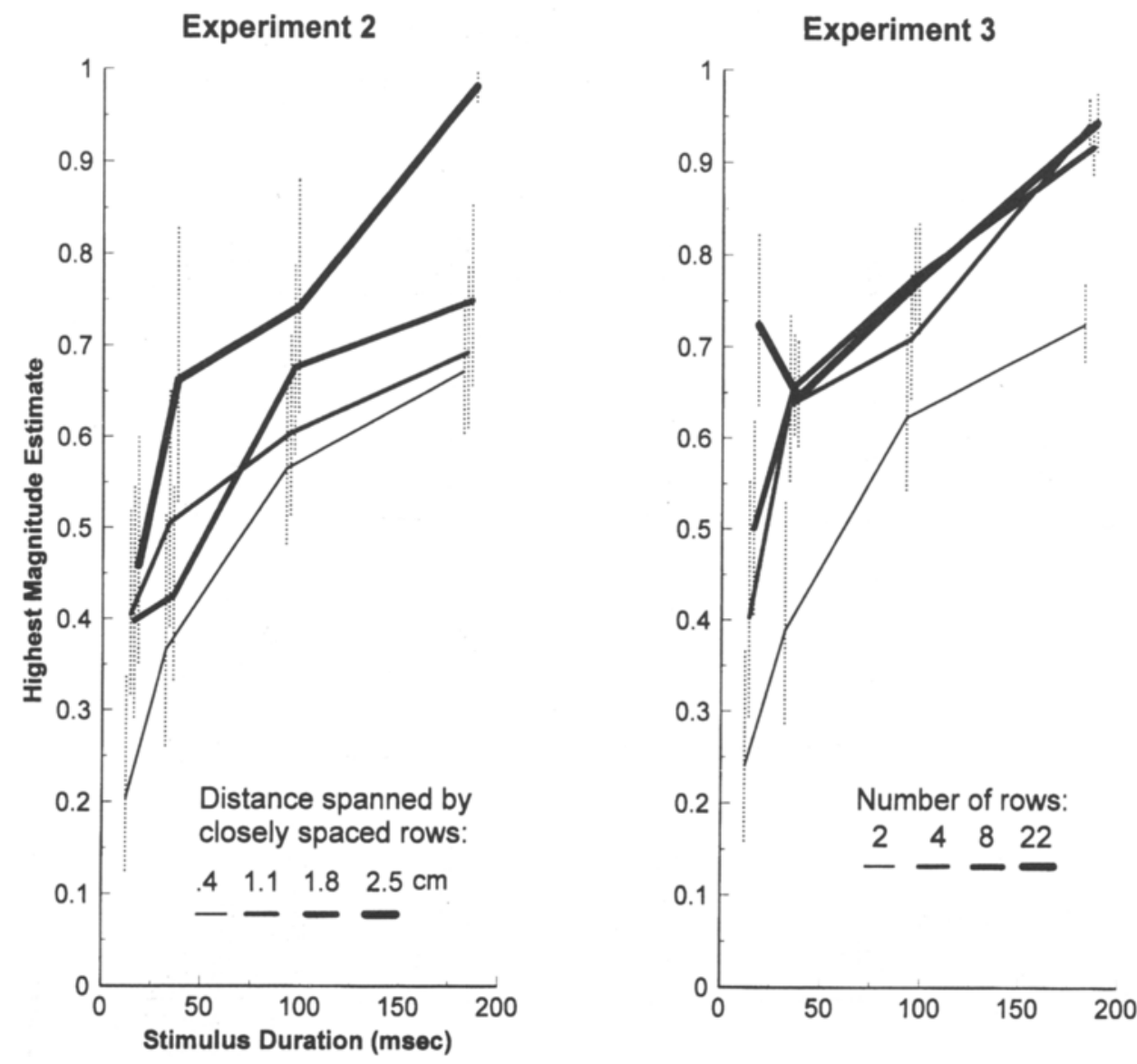

Figure 10. The highest magnitude estimates of goodness of motion obtained from the data of Experiment 2 (left panel) and Experiment 3 (right panel). The format is identical to that of Figure 7 (left panel).

Figure 10, left panel, with Figure 7, left panel]. A comparison of Figures 9 and 5 further reveals that subjects rated the percepts evoked at the shorter durations by multirow patterns more highly than those evoked by the two-row patterns. Second, the highest magnitude estimates increased with the distance over which the percepts were evoked $[F(3,27)=17.3, p<.0002]$. The impact of the 7-fold variation in the distance of skin traversed approximated $56 \%$ of that attained by the 14.6 -fold change in stimulus duration. This suggests that the highest magnitude estimate was affected to roughly the same extent by proportional changes in either parameter: That is, a 7fold increase in distance traversed or in stimulus duration over the ranges of values studied would increase the highest magnitude estimate about the same amount.

For the analysis described above, the highest magnitude estimates were extracted without consideration of the number of rows simultaneously activated. Examination revealed that $72 \%$ of the ISOI values at which the highest magnitude estimates were attained approximated one of the first seven integral fractions of stimulus duration. Thirty percent approximated the first integral fraction, attesting to the particularly smooth and continuous percepts evoked by the highly regular, step-locked transition of a single row to the next.

Additional analyses were undertaken to quantitatively evaluate the goodness of motion for different numbers of simultaneously active rows and for the impact of apparent velocity. To this end, three measures were extracted from the responses of each subject, as is illustrated in Figure 11 . These measures were the highest mean magnitude estimates within a window of $\pm 10 \mathrm{msec}$ around values of ISOI that were equal to one, one-half, and one-third times the stimulus duration. Magnitude estimates for fractions smaller than one third were not available for all four stimulus durations and, thus, were not included in the analyses. The values were analyzed to determine the effects of the number of rows simultaneously activated $(1,2$, or 3$)$, of the distance over which the movement was emulated $(0.4,1.1,1.8$, and $2.5 \mathrm{~cm})$, and of the apparent velocity of motion.

It was discovered that the number of rows simultaneously activated did not affect goodness of motion $[F(2,6)$ $=3.29, p>.10]$. In contrast, a significant effect of the distance traversed $[F(3,129)=3.87, p<.02]$ and of the distance $\times$ velocity interaction $[F(3,129)=5.76, p<.001]$ 


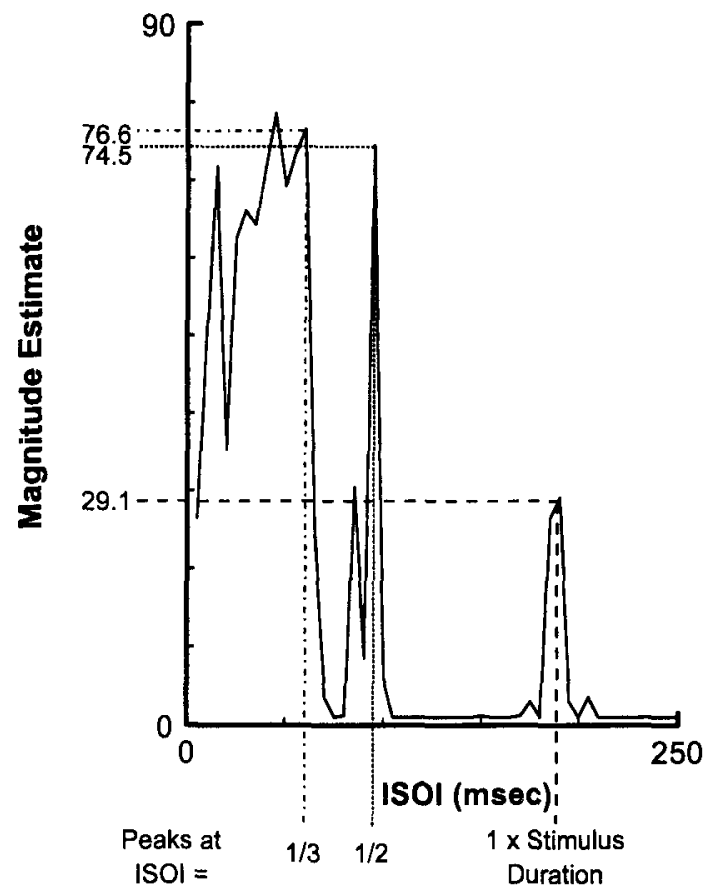

Figure 11. Geometric means of the magnitude estimates from Subject 224 when closely spaced stimuli were sequentially activated over $1.8 \mathrm{~cm}$ of skin and the duration of stimulation was 185 msec. Values marked on the $y$-axis are the maximum mean magnitude estimates within 10 -msec windows around interstimulus onset interval values equal to the first three integral fractions of the stimulus duration.

was detected. The distance $\times$ velocity interaction is graphically illustrated by Figure 8 , right panel, in which the regression lines for the four distances have been drawn. As is shown in the figure, goodness of motion only decreased with velocity for the shortest distance across the skin $(0.4 \mathrm{~cm})$. For longer distances, the magnitude estimates were numerically less, but not statistically so, for apparent velocities $<1 \mathrm{~cm} / \mathrm{sec}$. Thus, with only one exception, goodness of motion did not vary significantly with the number of rows simultaneously activated, the length of skin traversed, or the apparent velocity of motion.

We concluded that the translatory component of real movement was successfully emulated by the multirow patterns when values of ISOI approximated integral fractions of stimulus duration. In this situation, the rows rose and fell in a highly regular, step-locked rhythm so as to closely simulate a real constant-width object moving across the skin. The range of apparent velocities so attained (viz., $0.63-23.5 \mathrm{~cm} / \mathrm{sec}$; see Figure 8 , right panel) coincided with those useful for studying direction discrimination on the face $(0.5-32 \mathrm{~cm} / \mathrm{sec})$. Except over short distances across the skin $(0.4 \mathrm{~cm})$, the quality of the motion percepts remained essentially constant with variations in apparent velocity. These results differ notably from those obtained with only two successively activated rows (Experiment 1 ).
Specifically, with only two rows, percepts of motion were attained at apparent velocities that exceed the range over which human subjects extract information about direction of movement. Moreover, the percepts decreased in quality with increases in apparent velocity.

\section{EXPERIMENT 3 Constant Distance, Different Number of Rows}

\section{Method}

In Experiment 2, it was shown that the highest magnitude estimate of goodness of motion increased with the number of closely spaced rows that were sequentially activated. However, when only those temporal conditions that led to a smooth transition from one row to the next or to the simultaneous activation and translation of two (or three) rows were considered, no difference was detected for 10,16 , and 22 rows. Experiment 3 attempted to obtain additional insight into the impact of the number of stimuli on the goodness of motion. Specifically, to what extent was the improvement in the quality of the best motion attainable because of an increase in the number of rows, as opposed to a concomitant increase in distance across the skin?

As in Experiment 2, multiple equally spaced rows were sequentially activated (Figure 2C). The spacing, however, varied $(2.5,0.7$, 0.4 , and $0.12 \mathrm{~cm}$ ), such that $2,4,8$, or 22 rows were sequentially activated across the same cutaneous field of ca. $2.5 \mathrm{~cm}$ in length. Two possibilities were under investigation. First, assuming that information about the 2-row patterns (Experiment 1) and the multirow patterns (Experiment 2) was processed in a similar manner (an assumption comparable to that of past investigators; Kirman, 1975), goodness of motion was predicted to increase in a graded manner with the number of rows stimulated. This is because the increase in goodness of motion observed in Experiment 2 must have been due to an increase in the number of stimuli. The concomitant increase in the total distance would not have impacted goodness of motion (Experiment 1).

Alternatively, if one assumes that information about the two-row patterns (Experiment 1) and the multirow patterns (Experiment 2) was processed in different manners, goodness of motion may not increase in a graded manner with the number of rows stimulated. This is because the increase in goodness of motion observed in Experiment 2 may have been due solely to the increase in the total distance traversed.

\section{Results and Discussion}

Method-of-limits sessions. The ranges of ISOI values over which motion was perceived were grossly comparable with those observed for the corresponding or similar patterns in Experiments 1 and 2 (compare the right panel in Figure 3 with the left and middle panels, respectively). In addition to a significant effect of stimulus duration $[F(3,9)=33.9, p<.0002]$, a number of rows $X$ stimulus duration interaction was detected $[F(9,27)=3.11, p<.02]$ and accounted for the difference observed between the data obtained with two rows and those obtained with four or more rows. Although the values were not identical, the general similarity between the data obtained with two widely separated rows in Experiments 1 and 3 and between the data obtained with the multirow patterns in Experiments 2 and 3 attests to the reliability of findings across experiments. 
As in Experiments 1 and 2, motion was perceived for the lowest positive value $(5 \mathrm{msec})$. Gaps in the range of ISOI values, over which the percepts did not meet the subjects' criterion for motion, were identified when stimulus duration equaled 95 and $185 \mathrm{msec}$, but not as frequently as in Experiment 2 . The data from 3 of the 4 subjects exhibited gaps for a stimulus pattern of 22 rows. Two subjects' data showed gaps for the 8-row pattern, and one subject's data showed gaps for the 4-row pattern. None of the subjects' data showed gaps for the 2-row pattern. Similar findings were obtained with the 2-row and the 22-row patterns in Experiments 1 and 2, respectively, which again attests to the reliability of findings across experiments.

The number of optima marked by the subjects increased from 0.59 for the shortest duration stimuli to 1.56 for the longest duration stimuli, on average, independently of the number of rows sequentially activated. The frequency at which each value of ISOI was marked as an optimum is shown in Figure 4 (third row of plots). The distribu- tions are similar to those in Experiment 2; however, fewer total optima were marked.

Magnitude-estimation sessions. The intraclass correlation coefficient (mean $=.76$ ) confirmed a high degree of day-to-day consistency in the influence of the experimental variables on each subject's responses. The means of the magnitude estimates were plotted as functions of ISOI, as is shown in Figure 12. It is important to note that, as the number of rows distributed over the same distance was increased from two to four or more, the profile characterizing the magnitude estimate versus ISOI relationship became progressively taller and more skewed toward the $y$-axis (see the third and fourth columns of the plots in Figure 12). This implies that the quality of the motion percepts improved, but at the lower ISOI values.

As in Experiments 1 and 2, the results of the analyses of the highest, the lowest, and the range of ISOI values that supported percepts of motion were very similar to those of the analogous measures determined from the

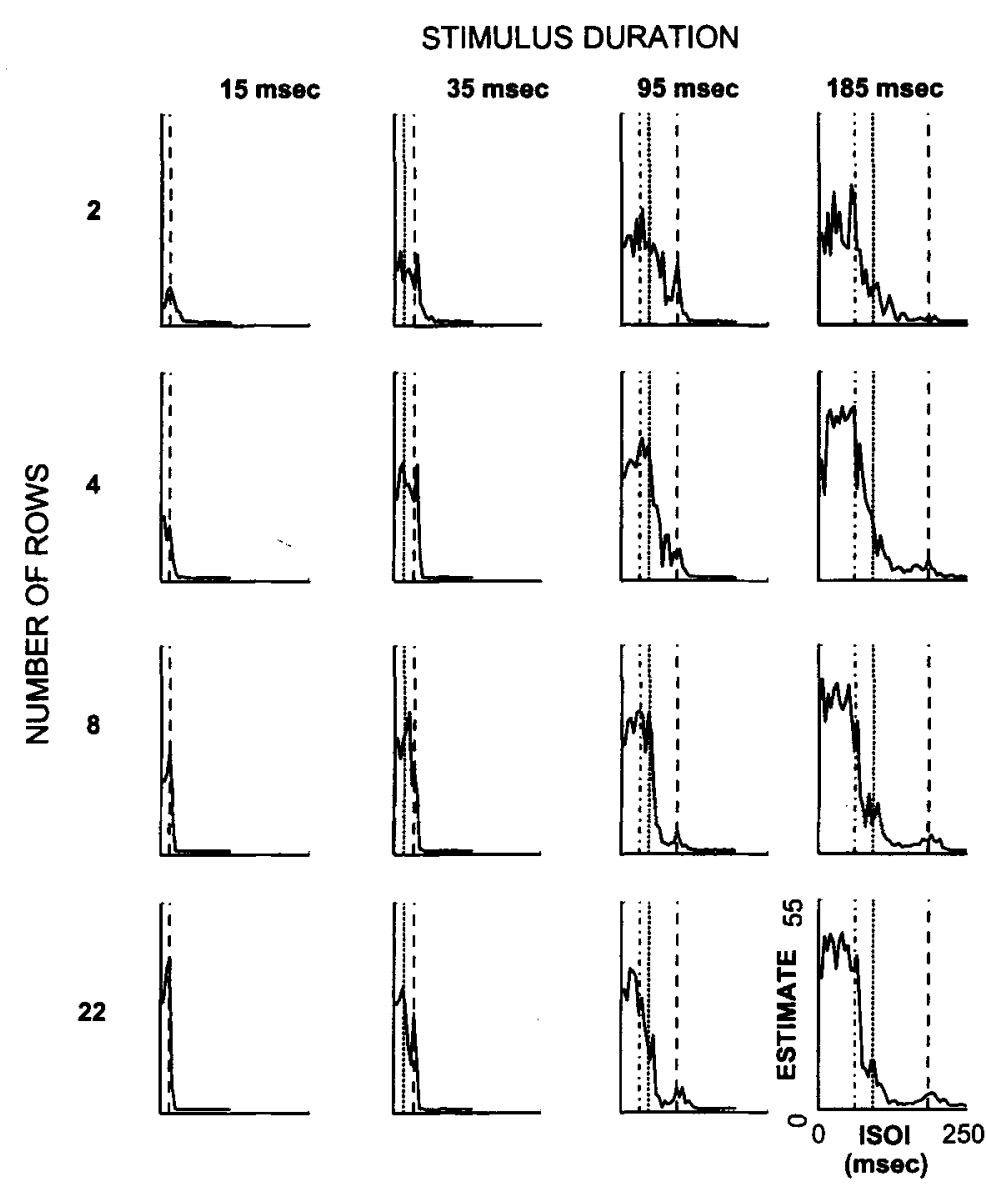

Figure 12. Geometric means of the magnitude estimates of Experiment 3, plotted as a function of interstimulus onset interval (ISOI) for each duration of stimulation (column of plots) and number of rows sequentially activated (row of plots). The format is identical to that of Figure 9. 

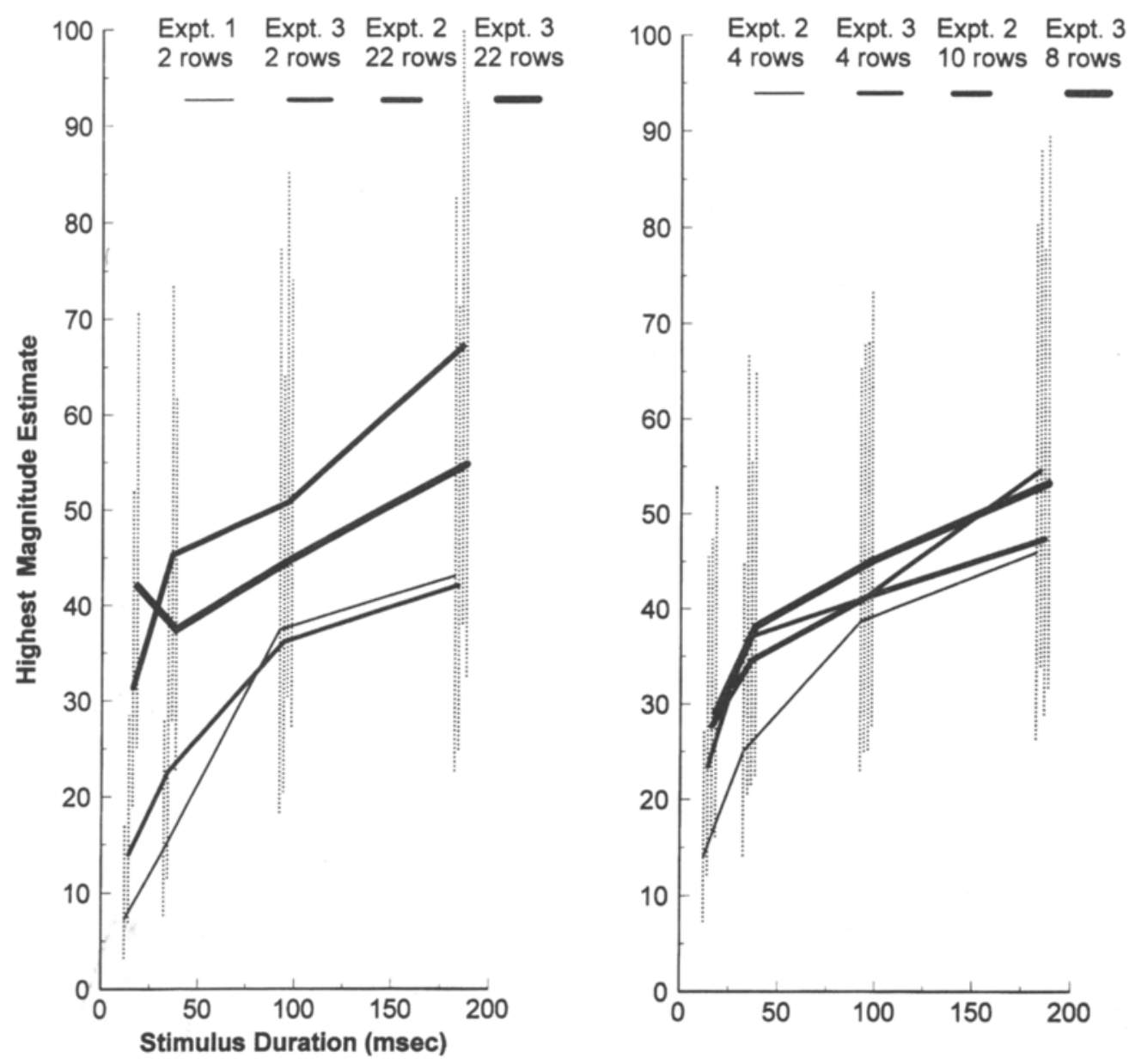

Figure 13. Comparison of the highest magnitude estimates obtained from the three experiments. (Left panel) Data from 2 rows separated by $2.5 \mathrm{~cm}$ and from 22 rows equally spaced over a total distance of $2.5 \mathrm{~cm}$. (Right panel) Data from 4 and 10 closely spaced rows (Experiment 2) and 4 and 8 rows distributed over a total distance of about $2.5 \mathrm{~cm}$ (Experiment 3). The format is identical to that of Figure 7 (left panel).

method-of-limits data (this topic is further discussed in Appendix B). As in Experiment 2, the highest magnitude estimate increased modestly with the 14.6-fold increase in stimulus duration [2.0-fold, compared with 2.2-fold in Experiment 2; $F(3,9)=7.85, p<.008$; Figure 10]. Although the highest magnitude estimate increased with the number of rows sequentially activated $[F(3,9)=6.40$, $p<.02$ ], the number of rows affected the measure in a graded manner for only the shortest stimulus duration $[F(9,27)=2.91, p<.015$, for the number of rows $\times$ stimulus duration interaction]. As is made evident by Figure 10 (right panel), for durations of activation $\geq 35 \mathrm{msec}$, maximum goodness of motion was very similar for the 4-, 8- and 22-row patterns but significantly greater than that for the 2-row pattern. Thus, we cannot rule out the possibility that the graded increase in goodness of motion with number of stimuli observed in Experiment 2 (see Figure 10, left panel) was due to the concomitant increase in the total distance traversed by the stimuli.
To investigate this question further, we first determined whether the subjects assigned the same numbers to the same stimulus pattern across experiments. The common patterns were 2 rows separated by $2.5 \mathrm{~cm}$ (Experiments 1 and 3 ) and 22 closely spaced rows distributed over a distance of $2.5 \mathrm{~cm}$ (Experiments 2 and 3; see Figure 2). Estimates of the highest magnitude estimates are shown in Figure 13 (left panel). Repeated-measures analyses confirmed that the mean estimate for the 22-row pattern exceeded that for the 2-row pattern $[F(3,9)=8.57$, $p<.05]$; however, the mean estimates for the same pattern across experiments were statistically comparable.

Second, we extracted those data from Experiment 2 and Experiment 3 that were obtained with stimulus patterns that were composed of about the same number of rows but were distributed over different distances. These included (1) 4 closely (Experiment 2) and 4 maximally (Experiment 3 ) spaced rows and (2) 10 closely spaced (Experiment 2 ) and 8 maximally spaced (Experiment 3 ) 
rows. Estimates of the highest magnitude estimates are shown in Figure 13 (right panel). If the total distance over which the stimuli were spaced was important, the numbers assigned to the member of each pair with the more widely spaced rows should have been greater. Although the means for the widely spaced stimuli were greater than those for the closely spaced stimuli, differences did not reach statistical significance $[F(3,9)=3.28$, $p>.07]$. Thus, the data suggest but do not provide convincing evidence that the distance over which multiple, sequentially activated stimuli are distributed contributes to the smoothness and continuity of apparent motion. The relative importance of the number of stimuli as opposed to the total distance across the skin cannot be resolved with the existing data.

\section{GENERAL DISCUSSION}

The results of the three experiments confirm and extend previous findings regarding percepts of motion that were evoked by discrete vibrotactile stimulation of the skin. In addition, the possibility that more than one mechanism underlies the percepts is suggested by the differential effects observed from the successive stimulation of only two, as opposed to multiple, sites. These differences are summarized below with the more general findings of the study.

Importance of the delay between the onset of stimulation of successive sites. The ISOI proved to be the most critical determinant of the presence rather than the absence of apparent motion. However, no single delay defined a temporal requirement. Rather, for the successive activation of two rows, both the highest value of ISOI for which motion was perceived and the value for which the quality was optimal increased with the duration for which each row stimulated the skin. The relationships between these values of ISOI and stimulus duration were relatively independent of the spatial separation between the two stimuli. Such relationships are characteristic of the apparent motion evoked by the successive delivery of two stimuli separated by distances much greater than those employed in the present study (see Kirman, 1974a; Sherrick, 1968; Sherrick \& Rogers, 1966). A new finding was that the motion percepts evoked by two stimuli decreased in quality with increases in the apparent velocity of stimulation.

For the successive activation of multiple $(\geq 4)$ closely spaced rows, the relationship between the optimal value of ISOI and stimulus duration changed: Multiple values of ISOI, which approximated integral fractions of stimulus duration, were associated with particularly good percepts of motion. For the condition in which the ISOI approximated the first three integral fractions of stimulus duration, one, two, or three simultaneously activated rows, respectively, translated across the skin, and goodness of motion remained essentially constant with changes in apparent velocity.
For the successive activation of either two or multiple rows, the lowest value of ISOI that supported percepts of motion approximated $5 \mathrm{msec}$ and was smaller than that reported by previous investigators (Kirman, 1974a, 1975, 1983; Sherrick, 1968; Sherrick \& Rogers, 1966). However, previous studies employing stimuli in close spatial proximity have not evaluated percepts that were evoked by positive values of ISOI less than $10 \mathrm{msec}$ (Kirman, 1974a, 1983), $12 \mathrm{msec}$ (Hill \& Bliss, 1968), or $20 \mathrm{msec}$ (Kirman, 1974b). With the exception of Kirman (1983), percepts of apparent motion have been observed at these short interstimulus delays. Hill and Bliss's subjects even reported apparent motion when airjet stimuli were presented simultaneously. Kirman's $(1975,1983)$ data indicate that higher values of ISOI are required if subjects are instructed to respond to clearly discernible forms in motion than would be required otherwise. Smaller values resulted in faster motion and indiscernible forms. Similarly, we have recently found that information about direction decreases with increases in apparent velocity beyond $6 \mathrm{~cm} / \mathrm{sec}$ (Essick et al., 1996). Thus, little association can be inferred between perceived smoothness and continuity, on the one hand, and clarity of stimulus direction, velocity, form, and shape, on the other (see also Sherrick \& Rogers, 1966).

Impact of the number of sites successively stimulated. The experiments showed that, as the number of vibrating rows sequentially activated on the face is increased from two to four or more, the quality of the motion percepts improves. However, it could not be determined to what extent the improvement was due to the increase in number of rows rather than to a concomitant increase in distance across the skin. The differences in the magnitude estimates for patterns with four or more than four rows, regardless of whether the rows were adjacent or distributed over the same constant distance, were too small and variable among subjects to make this distinction. The difficulty in making this distinction is not unique to the present work but has been reported by others (Kirman, 1975). Moreover, the impact of only one additional stimulus (i.e., a total of three) remains unclear. For example, Hill and Bliss (1968) showed that an increase in the number of airjet stimuli from two to three did not significantly affect either ratings of the motion quality or the ISOI values for which motion percepts are evoked.

The present experiments also demonstrated that, as the number of vibrating rows sequentially activated on the face is increased from two to four or more, the optimal values of ISOI shift to lower values. Kirman (1975) first observed this phenomenon and concluded that tactile information could be transferred more rapidly through apparent motion with multisite, rather than with two-site, stimulation. We showed, however, that, when the stimuli are very closely spaced, the apparent velocities supporting motion percepts are appreciably lower than those supporting motion percepts for two-stimulus patterns (cf. Figure 8). 
Impact of the duration of stimulation. For the successive activation of two or multiple rows, the duration of stimulation at each site consistently influenced the evocation of apparent motion. Specifically, both the range of ISOI values that supported percepts of motion and the magnitude estimates provided by the subjects increased with the duration for which each row stimulated the skin. This finding is in total agreement with the existing literature that describes an increased impressiveness or frequency of apparent motion in response to an increase in stimulus duration (Kirman, 1974a, 1974b, 1975; see also Gibson, 1968). However, in the present study, increases in stimulus duration had less than half the impact for multirow patterns than for the simplest two-row pattern (e.g., compare the third row of plots in Figure 9 with the third row of plots in Figure 5), which provides additional evidence that two different mechanisms may be at work.

Differences in the impact of four factors on percepts of apparent motion were identified for the successive activation of two as opposed to four or more stimulus sites. These factors were (1) the delay between the onsets of stimulation, (2) the apparent velocity of motion, (3) the total distance over which percepts were evoked, and (4) the duration that each site was stimulated. To further address whether more than one mechanism may underlie percepts of apparent motion, we sought neurophysiological evidence.

Peripheral neural mechanisms cannot account for percepts of apparent motion evoked by either tworow or multiple-row stimulus patterns. Responses of individual mechanoreceptors to stimuli provided by a device similar to the dense-array tactile stimulator (i.e., the OPTACON) have been studied in the monkey (Gardner, Hämäläinen, Palmer, \& Warren, 1989; Gardner \& Palmer, 1989; Gardner, Palmer, Hämäläinen, \& Warren, 1992).
Gardner and colleagues found that the predominate type of mechanoreceptor responding to these stimuli exhibited uniform sensitivity: One action potential per stimulus pulse was typically evoked, independently of the location of the probes in relation to the receptive field (RF) center. Moreover, the afferents did not sum inputs from more than one row in the RF. Assuming that similar responses were evoked by the dense-array tactile stimulator, essentially the same level (mean firing rate) and pattern of discharge, reflecting only the temporal frequency of vibrotactile stimulation, were evoked by all the stimuli employed in the present study. Thus, qualitative differences in the percepts (viz., percepts of smooth, continuous motion versus "bumpy" percepts of successively delivered, discrete stimuli) cannot be explained by the response evoked in the individual afferents for either tworow or multirow stimulus patterns.

We subsequently questioned whether the influence of changes in the spatial conditions on the percepts could be attributed to differences in the recruitment pattern of mechanoreceptor activation. A peripheral explanation implies that smooth, continuous motion could only be perceived when each successively activated row stimulated either the same RFs as those that were stimulated by the previous row or new RFs that contacted or overlapped those stimulated by the previous row. In the present work, percepts of smooth, continuous motion were reported for all of the spatial patterns employed; however, the separation between successively activated rows for some of the patterns exceeded the separation that one might predict would result in stimulation of adjacent mechanoreceptors along the path of motion.

To assess whether the influence of changes in the spatial conditions on the percepts could be attributed to differences in the recruitment pattern of mechanoreceptor

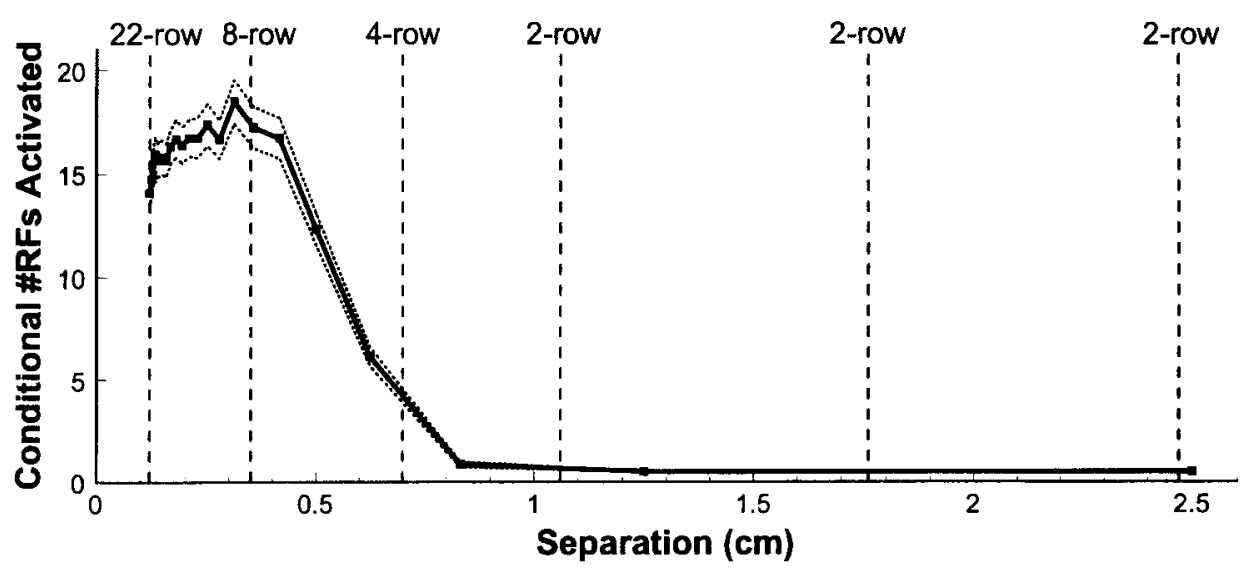

Figure 14. Results of simulations that model the sequential activation of mechanoreceptive afferents supplying the facial test site by the dense-array stimuli. The solid (dotted) line(s) indicate the mean ( \pm 1 standard error) number of receptive fields (RFs) activated by the stimulus, given that the RF was also activated at the previous position of the stimulus or spatially overlapped with an RF that was activated at the previous position. The abscissa represents the distance between successive positions of the stimulus. From left to right, the six vertical dashed lines indicate the separation between adjacent rows of the 22-row, 8-row, 4-row $($ spacing $=0.7 \mathrm{~cm}), 2$-row $($ separation $=1.1 \mathrm{~cm}), 2$-row $($ separation $=1.8 \mathrm{~cm})$, and 2-row (separation $=\mathbf{2 . 5} \mathrm{cm}$ ) patterns employed in the psychophysical experiments. 
activation, we conducted a computer simulation (see Appendix A). The sequential activation of the mechanoreceptive afferents that supplied the facial test site by the dense-array stimuli was modeled. The results are summarized in Figure 14. Shown are the means (solid line), $\pm 1 S E$ (dotted lines), of the number of RFs that (1) were activated by the stimulus and (2) were either activated themselves or overlapped with an RF that was activated at the previous position of the stimulus. The abscissa represents the distances between successive positions of stimulus delivery. If a spatially continuous pattern of mechanoreceptor activation is requisite for percepts of smooth motion, the relationship illustrated in Figure 14 suggests the following. First, percepts of smooth motion cannot be evoked by successively delivered stimuli that are separated by more than $0.8 \mathrm{~cm}$; positions that are separated by distances greater than this critical separation are not supplied by mechanoreceptors with spatially adjacent RFs. Second, the motion percepts evoked by successively delivered stimuli separated by less than $0.8 \mathrm{~cm}$ should not be equally smooth and continuous.

In contrast with the predictions of the model, percepts of smooth, continuous motion were attained with stimuli separated by distances appreciably greater than $0.8 \mathrm{~cm}$ : The six vertical dashed lines in Figure 14 indicate the separation between adjacent rows in the different patterns employed in the psychophysical experiments, for all of which percepts of smooth, continuous motion were attained. Moreover, goodness of motion was almost identical for stimuli separated by distances less than $0.8 \mathrm{~cm}$ for stimulus durations $\geq 35 \mathrm{msec}$ (see Figure 10, right panel, and Figure 12, plots in Rows 2-4). Thus, the influence of changes in the spacing and number of row stimuli on the percepts could not be attributed to differences in the recruitment pattern of mechanoreceptor activation.

Evidence for two central mechanisms subserving tactile motion perception. Gardner and colleagues demonstrated a fundamental role for central neural mechanisms in determining the qualitative nature of the percept evoked by a sequence of discrete tactile stimuli (Gardner et al., 1989; Gardner et al., 1992). Specifically, responses evoked in primate somatosensory cortical, motionsensitive neurons by the OPTACON stimuli were shown to differ from those evoked in the periphery. Pronounced oscillations in discharge, resulting from individual stimuli of $4 \mathrm{msec}$ duration, were observed when the pulses were applied slowly (i.e., ISOI $=40 \mathrm{msec}$ ). When examined by the experimenters, the individual tactile pulses could be resolved and the stimuli felt "bumpy." In contrast, when the pulses were applied rapidly (i.e., ISOI = $10 \mathrm{msec}$ ), the cortical neuron discharge was continuous and resembled that evoked by a natural stimulus object (e.g., a brush) moving across the skin. Moreover, the stimuli evoked percepts of smooth, continuous motion. It was concluded that the degree of modulation (i.e., periodic bursts versus continuous discharge) in the discharge pattern determined whether a sequence of discrete stim- uli or smooth motion was perceived. That individual primary somatosensory cortical neurons respond to appropriately timed sequences of multiple discrete stimuli and to real movement in a comparable manner implies that they are sensitive to, and process information about, the translatory component of real movement across the skin.

The question as to whether these same cortical neurons can signal the presence of movement in response to the successive delivery of only two discrete stimuli is unanswered. No study, to our knowledge, has demonstrated a continuous discharge in somatosensory, motion-sensitive cortical neurons, similar to that evoked by real movement across the RF, in response to the successive presentation of only two discrete stimuli. Two widely separated stimuli activate different populations of cortical neurons, and it has been suggested that percepts of apparent motion may arise from higher order central neural mechanisms that are sensitive to the rate at which and to the direction in which the activity shifts across the primary somatosensory cortex in response to their successive delivery (Costanzo \& Gardner, 1980; Sherrick \& Rogers, 1966). In the present study, it is likely that two stimuli separated by even the maximum separation fell within the RFs of many somatosensory cortical neurons (see Dreyer, Loe, Metz, \& Whitsel, 1975). Thus, the central neural basis for percepts of smooth apparent motion evoked by the successive activation of two discrete stimuli remains unclear.

Additional neurophysiological studies are needed to determine the minimum number and maximum separation of discrete stimuli for which responses, similar to those evoked by real movement across the skin, can be attained in primary somatosensory cortical neurons. These spatial conditions could be interpreted as defining objective criteria by which stimulus sequences can be categorized as resembling the translatory component of real movement across the skin. The present work makes it clear that subjective, percept-based criteria are not useful in this regard. Accordingly, percepts of smooth apparent motion that are evoked by stimulus sequences that do not meet the objective criteria would necessarily reflect the operation of higher order central neural mechanisms.

\section{REFERENCES}

BARLOW, S. M. (1987). Mechanical frequency detection thresholds in the human face. Experimental Neurology, 96, 253-261.

Chen, C. C., Essick, G. K., Kelly, D. G., Young, M. G., Nestor, J. M., \& MAsse, B. (1995). Gender-, side- and site-dependent variations in perioral spatial resolution. Archives of Oral Biology, 40, 539-548.

Cholewiak, R. W., \& Collins, A. A. (1990). The effects of a plasticfilm covering on vibrotactile pattern perception with the Optacon. Behavior Research Methods, Instruments, \& Computers, 22, 21-26.

Collins, A. A., \& Gescheider, G. A. (1989). The measurement of loudness in individual children and adults by absolute magnitude estimation and cross-modality matching. Journal of the Acoustical Society of America, 85, 2012-2021.

Costanzo, R., \& Gardner, E. P. (1980). A quantitative analysis of responses of direction-sensitive neurons in somatosensory cortex of awake monkey. Journal of Neurophysiology, 43, 1319-1341.

Dreyer, D. A., Loe, P. R., Metz, C. B., \& Whitsel, B. L. (1975). Rep- 
resentation of head and face in postcentral gyrus of the macaque. Journal of Neurophysiology, 38, 714-734.

Edin, B. B., Essick, G. K., Trulsson, M., \& Olsson, K. Å. (1995). Receptor encoding of moving tactile stimuli in humans: I. Temporal pattern of discharge of individual low-threshold mechanoreceptors. Journal of Neuroscience, 15, 830-847.

Essick, G. K. (1992). Comprehensive clinical evaluation of perioral sensory function. Oral \& Maxillofacial Surgery Clinics of North America, 4, 503-526.

Essick, G. K., Afferica, T., Aldershof, B., Nestor, J., Kelly, D., \& WHITSEL, B. (1988). Human perioral directional sensitivity. Experimental Neurology, 100, 506-523.

Essick, G. K., Dolan, P. J., Turvey, T. A., Kelly, D. G., \& Whitsel, B. L. (1990). Effects of trauma to the mandibular nerve on human perioral directional sensitivity. Archives of Oral Biology, 35, 785-794.

Essick, G. K., Franzén, O., \& Whitsel, B. L. (1988). Discrimination and scaling of velocity of stimulus motion across the skin. Somatosensory \& Motor Research, 6, 21-40.

Essick, G. K., MCGuire, M., Joseph, A., \& Franzén, O. (1992). Characterization of the percepts evoked by discontinuous motion over the perioral skin. Somatosensory \& Motor Research, 9, 175-184.

Essick, G. K., Rath, E. M., Kelly, D. G., James, A., \& Murray, R. A. (1996). A novel approach for studying direction discrimination. In O. Franzén, R. Johansson, \& L. Terenius (Eds.), Somesthesis and the neurobiology of the somatosensory cortex (pp. 59-72). Basel: Birkhäuser Verlag.

Essick, G. K., Whitsel, B., Dolan, P., \& Kelly, D. (1989). Effects of traverse length on human perioral directional sensitivity. Journal of the Neurological Sciences, 93, 175-190.

Gardner, E. P., Hämäläinen, H. A., Palmer, C. I., \& Warren, S. (1989). Touching the outside world: Representation of motion and direction within primary somatosensory cortex. In J. Lund (Ed.), Sensory processing in the mammalian brain: Neural substrates and experimental strategies (pp. 49-66). New York: Oxford University Press.

Gardner, E. P., \& PAlmer, C. I. (1989). Simulation of motion on the skin: I. Receptive fields and temporal frequency coding by cutaneous mechanoreceptors of OPTACON pulses delivered to the hand. Journal of Neurophysiology, 62, 1410-1436.

Gardner, E. P., Palmer, C. I., Hämäläinen, H. A., \& Warren, S. (1992). Simulation of motion on the skin: V. Effect of stimulus temporal frequency on the representation of moving bar patterns in primary somatosensory cortex of monkeys. Journal of Neurophysiology, 67, 37-63.

GESCHEIDER, G. A. (1988). Psychophysical scaling. Annual Review of Psychology, 39, 169-200.

GiBSON, R. H. (1968). Electrical stimulation of pain and touch. In D. R. Kenshalo (Ed.), The skin senses (pp. 223-261). Springfield, IL: Thomas.

HiLL, J. W., \& BLISs, J. C. (1968). Perception of sequentially presented tactile point stimuli. Perception \& Psychophysics, 4, 289-295.

Hollins, M., Delemos, K. A., \& Goble, A. K. (1991). Vibrotactile adaptation on the face. Perception \& Psychophysics, 49, 21-30.

Johansson, R. S., Trulsson, M., Olsson, K. A., \& WestberG, K.-G. (1988). Mechanoreceptor activity from the human face and oral mucosa. Experimental Brain Research, 72, 204-208.

Johansson, R. S., \& VAllbo, Å. B. (1983). Tactile sensory coding in the glabrous skin of the human hand. Trends in Neuroscience, 6, 27-32.

Kirman, J. H. (1974a). Tactile apparent movement: The effect of interstimulus onset interval and stimulus duration. Perception \& Psychophysics, 15, 1-6.

KIRMaN, J. H. (1974b). Tactile apparent movement: The effect of number of stimulators. Journal of Experimental Psychology, 103, 1175-1180.

KIRMAN, J. H. (1975). The effect of number of stimulators on the optimal interstimulus onset interval in tactile apparent movement. Perception \& Psychophysics, 17, 263-267.

Kirman, J. H. (1983). Tactile apparent movement: The effects of shape and type of motion. Perception \& Psychophysics, 34, 96-102.

KoCH, G. G. (1985). Intraclass correlation coefficient. In S. L. Kotz \& N. L. Johnson (Eds.), Encyclopedia of statistical sciences (pp. 212217). New York: Wiley.
MyERS, J. L. (1966). Fundamental of experimental design. Boston: Allyn and Bacon.

ROGERS, R. (1964). Apparent tactual movement: An experimental study. Unpublished senior thesis, Princeton University.

SHERRICK, C. E. (1968). Studies of apparent tactual movement. In D. R. Kenshalo (Ed.), The skin senses (pp. 331-343). Springfield, IL: Thomas.

Sherrick, C. E., Cholewiak, R. W., \& Collins, A. A. (1990). The localization of low- and high-frequency vibrotactile stimuli. Journal of the Acoustical Society of America, 88, 169-179.

SHERRICK, C. E., \& Rogers, R. (1966). Apparent haptic movement. Perception \& Psychophysics, 1, 175-180.

Sumby, W. H. (1955). An experimental study of vibrotactile apparent motion. Unpublished master's thesis, University of Virginia, Charlottesville.

Szaniszlo, J. A., Essick, G. K., \& Kelly, D. G. (1992). Spatial/ temporal requirements for tactile motion perception. Journal of Dental Research (Special issue), 71, 197.

\section{APPENDIX A \\ Simulation of the Sequential Activation of Mechanoreceptive Afferents}

First, RF size and density were estimated. In accord with Johansson et al. (1988) and Edin et al. (1995), RF size was assumed to be distributed log normally with the mean equal to $0.17 \mathrm{~cm}^{2}$ and the standard deviation of the $\log$ area equal to 0.13 . Moreover, RF size was constrained to be between 0.02 and $1.18 \mathrm{~cm}^{2}$. The minimum RF density was estimated to be 12 units $/ \mathrm{cm}^{2}$ on the basis of the following: (1) On the glabrous hand, peripheral innervation density and the two-point discrimination threshold vary inversely (Johansson \& Vallbo, $1983)$; (2) the vermilion is innervated as densely as the fingertip, at ca. 240 units $/ \mathrm{cm}^{2}$ (Johansson et al., 1988; Johansson \& Vallbo, 1983); (3) the two-point perception threshold is 6.6 times greater on the test site than on the vermilion (Chen et al., $1995)$; (4) the slowly adapting mechanoreceptors in the perioral region equal in number or outnumber the fast adapting mechanoreceptors two to one (Edin et al., 1995; Johansson et al., 1988); and (5) it is likely that only the fast-adapting mechanoreceptors are activated by the dense-array stimuli (see Essick et al., 1996). In addition, the RFs were assumed to be circular and their centers distributed randomly.

Second, the recruitment pattern of activation that was generated by edges contacting the skin from 1 to 21 equally spaced positions across a distance of $2.5 \mathrm{~cm}$ was simulated with MATLAB software (The MathWorks, Inc., 24 Prime Park Way, Natick, MA 01760). The width of the edge perpendicular to the direction of motion was assumed to be $1.3 \mathrm{~cm}$. Stimulus duration was assumed to be equal to ISOI (this simulated the condition of temporally uninterrupted stimulation to successive positions within the test field). The simulation was repeated 100 times for each number of jumps. A new collection of RFs was generated for each simulation. Results are shown in Figure 14.

The conclusion, that the impact of changes in the spatial conditions on the percepts cannot be attributed to differences in the recruitment pattern of mechanoreceptor activation (see text), depends on the validity of the assumptions regarding RF size and density. To determine how critical these assumptions are, we conducted additional simulations. The critical separation (i.e., the maximum separation between successive positions supplied by mechanoreceptors with spatially adjacent RFs) was found to be independent of innervation density over the range evaluated (6-36 units $/ \mathrm{cm}^{2}$ ) and increased only slightly (to $1.25 \mathrm{~cm}$ ), with 
a tripling of the mean of the distribution of RF areas. Thus, the conclusion is valid for broad but reasonable ranges of RF size and innervation density.

\section{APPENDIX B \\ Similarity of Results From Use of the Method-of-Limits and Magnitude-Estimation Approaches}

The two protocols led to remarkably consistent results regarding the percepts evoked.

1. The results of the analyses of the highest, the lowest, and the range of ISOI values that supported motion percepts were similar to the results for the analogous measures extracted from the method-of-limits data, particularly given the arbitrary criteria by which the limits were defined from the magnitudeestimation data.

2. The values of ISOI that a subject identified as optimal for achieving particularly good percepts of motion during descending or ascending series of stimuli did not necessarily correspond to the maxima of his(her) magnitude-estimation data. However, the two approaches for identifying optimal conditions provided comparable results in a statistical sense. For example, if one compares the profile of the frequency at which each value of ISOI was identified as an optimal value (Figure 4 ) with the profiles describing the magnitude estimate versus ISOI relationship (Figures 5, 9, and 12), a striking correspondence of the shapes is noted. It is particularly convincing for Experiment 2 (compare the second row of plots in Figure 4 with any row of plots in Figure 9).

3. Gaps in the range of ISOI values, over which the percepts did not meet subjects' criteria for motion, coincided in large part with those intervals over which subjects assigned very low numerical estimates of goodness. This is particularly evident in the data for Experiment 2. Specifically, during the method of limits, gaps were consistently identified for values of ISOI resulting in oscillation of the number of simultaneously activated rows from one and two. These values are precisely those for which subjects assigned numerical ratings approximating 1 during sessions employing magnitude estimation (see Figure 9).

Thus, we conclude that the information provided by the two experimental approaches provided consistent information about the impact of spatial and temporal stimulus parameters on percepts of apparent motion. The decided advantage of the magnitude-estimation procedure was that information about the relative goodness of motion was directly obtained.

(Manuscript received May 15, 1995; revision accepted for publication June 18, 1997.) 